\title{
Fourth Lepton Family is Natural in Technicolor
}

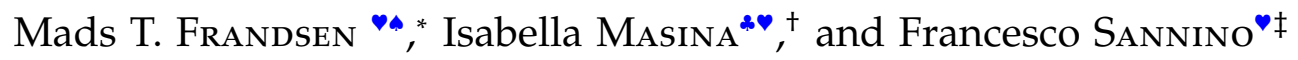 \\ - $C P^{3}$ - Origins, IFK \& IMADA, University of Southern Denmark, \\ Campusvej 55, DK-5230 Odense M, Denmark $\$$ \\ - Rudolf Peierls Centre for Theoretical Physics, University of Oxford, \\ 1 Keble Road, Oxford OX1 3NP, United Kingdom. \\ - Dip. di Fisica dell'Università degli Studi di Ferrara and INFN Sez. di Ferrara, \\ Via Saragat 1, I-44100 Ferrara, Italy
}

\begin{abstract}
Imagine to discover a new fourth family of leptons at the Large Hadron Collider (LHC) but no signs of an associated fourth family of quarks. What would that imply? An intriguing possibility is that the new fermions needed to compensate for the new leptons gauge anomalies simultaneously address the big hierarchy problem of the Standard Model. A natural way to accomplish such a scenario is to have the Higgs itself be composite of these new fermions. This is the setup we are going to investigate in this paper using as a template Minimal Walking Technicolor. We analyze a general heavy neutrino mass structure with and without mixing with the Standard Model families. We also analyze the LHC potential to observe the fourth lepton family in tandem with the new composite Higgs dynamics. We finally introduce a model uniting the fourth lepton family and the technifermion sector at higher energies.
\end{abstract}

\footnotetext{
$\S$ Centre of Excellence for Particle Physics Phenomenology dedicated to the understanding of the Origins of Mass in the universe. This is the new affiliation from September 1st 2009.

*Electronic address: toudal@ifk.sdu.dk

${ }^{\dagger}$ Electronic address: masina@fe.infn.it

‡Electronic address: $\overline{\text { sannino@ifk.sdu.dk }}$
} 


\section{Contents}

I. Introduction

II. A natural fourth family of leptons at the TeV-scale
A. Heavy leptons with an exact flavor symmetry
4
B. Promiscuous heavy leptons
C. Heavy Leptons in Minimal Walking Technicolor models

III. Parameter space constraints
A. Limits from LEP and Tevatron
B. Direct constraints on mixing
C. Indirect constraints from EW precision data
D. Cosmological constraints

IV. LHC phenomenology 16
A. Production and decay of the new leptons
16
B. Collider signatures of heavy leptons with an exact flavor symmetry
C. Collider Signatures of Promiscuous Heavy Leptons

Note added

A. Full mixing

B. MWT effective Lagrangian

C. EW parameters

D. Decay widths of the heavy leptons 


\section{INTRODUCTION}

If the LHC discovers a new fourth family of leptons but no associated quarks, what would that imply? Either the associated quarks are much heavier than the electroweak scale or a new set of fermions are needed to account for the 4th family lepton induced gauge anomalies. The new fermions could address directly the big hierarchy problem of the SM if their dynamics leads to a composite Higgs scenario. This is the setup we are going to investigate in this paper using as a template Minimal Walking Technicolor (MWT) [1].

To investigate the phenomenology of such a theory we must first discuss the electromagnetic neutral lepton sector. We will consider a general mass structure for the fourth neutrino and both the case of mixing and no mixing with the SM neutrinos. We will also summarize the (in)direct phenomenological constraints. We then analyze the interplay of the composite Higgs sector with the new lepton family at the LHC.

We will study the production and decay of the new leptons in proton - proton collisions which is relevant to select the LHC signatures for the discovery of these new leptons. We show that the composite Higgs structure can affect and differentiate the final signatures with respect to the case in which the Higgs is elementary. The bottom line is that one can experimentally determine if the fourth family is associated to a composite Higgs sector.

We then move on to a more general framework in which the fourth family of leptons, together with the technicolor sector unites in a second $S U(2) \times U(1)$ gauge group. This model has a number of interesting features and reduces to the one investigated above when the mass scale of the new gauge bosons is sufficiently larger than the electroweak one.

\section{A NATURAL FOURTH FAMILY OF LEPTONS AT THE TEV-SCALE}

In the $\mathrm{SM}$, the three lepton families, $\ell=e, \mu, \tau$, belong to the following representations of the gauge group $S U(3)_{c} \times S U(2)_{L} \times U(1)_{Y}$ :

$$
L_{\ell}=\left(v_{\ell L} \ell_{L}\right)^{T} \sim(1,2,-1 / 2) \quad, \quad \ell_{R} \sim(1,1,-1)
$$


where the chirality projectors $P_{L}=\left(1-\gamma_{5}\right) / 2$ and $P_{R}=\left(1+\gamma_{5}\right) / 2$ have been introduced and the relation $Q=T_{3}+Y$ has been adopted in order to define the hypercharge with respect to the electric charge. The neutral and charged current interactions of the SM leptons are then respectively accounted for by the Lagrangian terms:

$$
\begin{aligned}
& \mathcal{L}_{N C}=\frac{g}{\cos \theta_{W}}\left(\frac{1}{2} \bar{v}_{L} \gamma^{\mu} v_{L}-\frac{1}{2} \bar{\ell}_{L} \gamma^{\mu} \ell_{L}+\sin ^{2} \theta_{W} \bar{\ell} \gamma^{\mu} \ell\right) Z_{\mu}+e \bar{\ell} \gamma^{\mu} \ell A_{\mu} \\
& \mathcal{L}_{C C}=\frac{g}{\sqrt{2}} \bar{\ell}_{L} \gamma^{\mu} v_{L} W_{\mu}^{-}+\text {h.c. }
\end{aligned}
$$

where $\ell=\ell_{L}+\ell_{R}$ and $\theta_{W}$ is the Weinberg angle. Experimentally it has been observed that at least two of the SM neutrinos have a small mass, not larger than the eV-scale [2]. In the following, we will account for the light neutrino masses and mixings by means of an effective Majorana mass term, namely we add to the SM Lagrangian a dimension-5 non-renormalizable operator. Such a minimal extension of the SM is often referred to as the $3 v$-SM.

Our aim here is to study the phenomenology of an additional heavy lepton family, with masses about the $\mathrm{TeV}$-scale. Thus, we add to the $3 v$-SM matter content a 4th-family of leptons - for which we introduce the $\zeta$-flavor - composed by a lepton doublet, a charged lepton singlet and a gauge singlet:

$$
L_{\zeta}=\left(v_{\zeta_{L}} \zeta_{L}\right)^{T} \sim(1,2,-1 / 2) \quad, \quad \zeta_{R} \sim(1,1,-1), \quad v_{\zeta_{R}} \sim(1,1,0)
$$

The $\zeta$-charged lepton, $\zeta=\zeta_{L}+\zeta_{R}$, will have a Dirac mass term like the other three charged leptons of the SM, but large enough to avoid conflict with the experimental limits (more on this later). We work in the basis in which the $4 \times 4$ charged lepton mass matrix is diagonal.

\section{A. Heavy leptons with an exact flavor symmetry}

By imposing an exactly conserved new $\zeta$-lepton number we forbid, in this section, the mixing between the $\zeta$-neutrino and the three light neutrinos of the SM. The Lagrangian can be split as $\mathcal{L}=\mathcal{L}_{S M}+\mathcal{L}_{\zeta}$. 
The Lagrangian mass terms we take for the $\zeta$-sector reads:

$$
\mathcal{L}_{\zeta}^{\text {mass }}=-m_{\zeta} \bar{\zeta} \zeta-\frac{1}{2}\left[\left(\overline{v_{\zeta L}} \overline{\left(v_{\zeta R}\right)^{c}}\right)\left(\begin{array}{cc}
0 & m_{D} \\
m_{D} & m_{R}
\end{array}\right)\left(\begin{array}{c}
\left(v_{\zeta L}\right)^{c} \\
v_{\zeta R}
\end{array}\right)+\text { h.c. }\right],
$$

Diagonalizing the neutrino mass matrix above, we obtain two independent Majorana eigenstates, $N_{1}$ and $N_{2}$, with real and positive masses, $M_{1}$ and $M_{2}$ (for convention $M_{1} \leq$ $\left.M_{2}\right)$,

$$
M_{1}=\frac{m_{R}}{2}\left(\sqrt{1+4 \frac{m_{D}^{2}}{m_{R}^{2}}}-1\right), \quad M_{2}=\frac{m_{R}}{2}\left(\sqrt{1+4 \frac{m_{D}^{2}}{m_{R}^{2}}}+1\right),
$$

which are related to the original Dirac and Majorana masses according to:

$$
M_{1} M_{2}=m_{D}^{2}, \quad M_{2}-M_{1}=m_{R} .
$$

The $\zeta$-neutrino chiral states will be an admixture of the two Majorana eigenstates $N_{1}$ and $\mathrm{N}_{2}$ :

$$
\left(\begin{array}{c}
v_{\zeta L} \\
\left(v_{\zeta R}\right)^{c}
\end{array}\right)=\left(\begin{array}{cc}
i \cos \theta & \sin \theta \\
-i \sin \theta & \cos \theta
\end{array}\right)\left(\begin{array}{c}
P_{L} N_{1} \\
P_{L} N_{2}
\end{array}\right) \quad, \quad \tan 2 \theta=\frac{2 m_{D}}{m_{R}} .
$$

In the limit $m_{D} \ll m_{R}$ the seesaw mechanism would be at work (leading to $M_{1} \sim m_{D}^{2} / m_{R}$, $\left.M_{2} \sim m_{R}, v_{\zeta_{L}} \sim i P_{L} N_{1},\left(v_{\zeta R}\right)^{c} \sim P_{L} N_{2}\right)$. Here however we are more interested in the situation $m_{D} \sim m_{R}$, in which both Majorana neutrinos have a mass below the TeV-scale and hence have a large $S U(2)$-active component.

The neutral current interaction of the $\zeta$-leptons in terms of the heavy neutrino Majorana mass eigenstates reads:

$$
\mathcal{L}_{\zeta}^{N C}=\frac{g}{\cos \theta_{W}}\left(\frac{1}{2} \overline{v_{\zeta_{L}}} \gamma^{\mu} v_{\zeta_{L}}-\frac{1}{2} \overline{\zeta_{L}} \gamma^{\mu} \zeta_{L}+\sin ^{2} \theta_{W} \bar{\zeta} \gamma^{\mu} \zeta\right) Z_{\mu}+e \bar{\zeta} \gamma^{\mu} \zeta A_{\mu}
$$

where

$$
\overline{\nu_{\zeta_{L}}} \gamma^{\mu} v_{\zeta_{L}}=-\frac{\cos ^{2} \theta}{2} \bar{N}_{1} \gamma^{\mu} \gamma_{5} N_{1}-\frac{\sin ^{2} \theta}{2} \bar{N}_{2} \gamma^{\mu} \gamma_{5} N_{2}+i \cos \theta \sin \theta \bar{N}_{2} \gamma^{\mu} N_{1}
$$

The interaction of the $Z$ with a couple of $N_{1}$ or $N_{2}$ is axial, while the one with two different 
$N_{i}$ is a vector interaction. As for the charged current:

$$
\mathcal{L}_{\zeta}^{C C}=\frac{g}{\sqrt{2}} W_{\mu}^{-} \bar{\zeta}_{L} \gamma^{\mu}\left(i \cos \theta P_{L} N_{1}+\sin \theta P_{L} N_{2}\right)+\text { h.c. }
$$

The Dirac mass can be written in terms of the Yukawa coupling $y_{\zeta}$ and the Higgs vacuum expecation value $v$ as $m_{D}=y_{\zeta} v / \sqrt{2}$. Hence the interaction of the new neutrinos with the Higgs field reads:

$$
\begin{aligned}
\mathcal{L}_{\zeta}^{H} & =-\frac{m_{D}}{v}\left(\overline{v_{\zeta_{R}}} v_{\zeta_{L}}+\overline{\nu_{\zeta_{L}}} v_{\zeta_{R}}\right) H \\
& =-\frac{m_{D}}{v}\left[\cos \theta \sin \theta\left(\bar{N}_{1} N_{1}+\bar{N}_{2} N_{2}\right)-i\left(\cos ^{2} \theta-\sin ^{2} \theta\right) \bar{N}_{1} \gamma_{5} N_{2}\right] H .
\end{aligned}
$$

\section{B. Promiscuous heavy leptons}

In this section we consider the possibility that the new heavy leptons mix with the SM leptons. For clarity of presentation we assume that the heavy neutrinos mix only with one SM neutrino of flavor $\ell(\ell=e, \mu, \tau)$ while we present the general case in appendix A. The entries of the mass matrix are:

$$
-\mathcal{L}=\frac{1}{2}\left(\overline{v_{\ell L}} \overline{v_{\zeta L}} \overline{\left(v_{\zeta R}\right)^{c}}\right)\left(\begin{array}{ccc}
O(e V) & O(e V) & m^{\prime} \\
O(e V) & O(e V) & m_{D} \\
m^{\prime} & m_{D} & m_{R}
\end{array}\right)\left(\begin{array}{c}
\left(v_{\ell L}\right)^{c} \\
\left(v_{\zeta L}\right)^{c} \\
v_{\zeta R}
\end{array}\right)+\text { h.c. . }
$$

The measured values of the light neutrino masses suggest the entries of the upper $2 \times 2$ block to be of $O(\mathrm{eV})$ while the remaining entries are expected to be at least of the order of the electroweak energy scale. Given such a hierarchical structure and up to small corrections of $O\left(e V / M_{1,2}\right) \lesssim 10^{-11}$, one obtains the following form for the unitary matrix which diagonalises eq.(11):

$$
\left(\begin{array}{c}
v_{\ell L} \\
v_{\zeta L} \\
\left(v_{\zeta R}\right)^{c}
\end{array}\right)=V\left(\begin{array}{c}
P_{L} N_{0} \\
P_{L} N_{1} \\
P_{L} N_{2}
\end{array}\right), V=\left(\begin{array}{ccc}
\cos \theta^{\prime} & i \cos \theta \sin \theta^{\prime} & \sin \theta \sin \theta^{\prime} \\
-\sin \theta^{\prime} & i \cos \theta \cos \theta^{\prime} & \sin \theta \cos \theta^{\prime} \\
0 & -i \sin \theta & \cos \theta
\end{array}\right) \text {. }
$$


$N_{0,1,2}$ are the new (Majorana) mass eigenstates and

$$
\tan \theta^{\prime}=\frac{m^{\prime}}{m_{D}}, \quad \tan 2 \theta=2 \frac{m_{D}^{\prime}}{m_{R}}, \quad m_{D}^{\prime 2} \equiv m_{D}^{2}+m^{\prime 2} .
$$

The light neutrino $N_{0}$ has a mass of $O(e V)$. Up to corrections of $O(e V)$, the heavy neutrinos $N_{1,2}$ have masses given by:

$$
M_{1}=\frac{m_{R}}{2}\left(\sqrt{1+4 \frac{m_{D}^{\prime 2}}{m_{R}^{2}}}-1\right), \quad M_{2}=\frac{m_{R}}{2}\left(\sqrt{1+4 \frac{m_{D}^{\prime 2}}{m_{R}^{2}}}+1\right) \text {. }
$$

In fig. 11 we display $M_{1}$ and $M_{2}$ as a function of $m_{R}$ for three representative values of $m_{D}^{\prime}$ from top to bottom $m_{D}^{\prime}=\{170,100,70\} \mathrm{GeV}$. The smaller $m_{R}$ is the more the neutrinos $N_{1}$ and $N_{2}$ become (the two Weyl components of) a Dirac state.

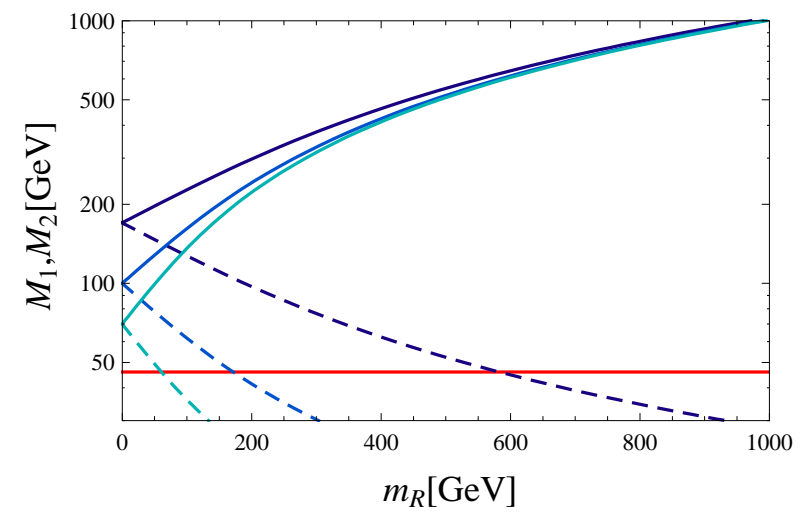

FIG. 1: $M_{1}$ (dashed-lines) and $M_{2}$ (solid-lines) as functions of $m_{R}$ for three representative values (from top to bottom) of $m_{D}^{\prime}=\{170,100,70\} \mathrm{GeV}$. The red (solid) horizontal line marks the value $M_{Z} / 2$.

Including the neutrino of flavor $\ell$, the fermion current in Eq. (9) is replaced with

$$
\begin{aligned}
\bar{v}_{\ell L} \gamma^{\mu} v_{\ell L}+\bar{v}_{\zeta_{L}} \gamma^{\mu} v_{\zeta_{L}} & =-\frac{1}{2} \bar{N}_{0} \gamma^{\mu} \gamma_{5} N_{0}-\frac{\cos ^{2} \theta}{2} \bar{N}_{1} \gamma^{\mu} \gamma_{5} N_{1}-\frac{\sin ^{2} \theta}{2} \bar{N}_{2} \gamma^{\mu} \gamma_{5} N_{2} \\
& +i \cos \theta \sin \theta \bar{N}_{2} \gamma^{\mu} N_{1},
\end{aligned}
$$

while the charged current terms in the Lagrangian, Eq. (10), become

$$
\mathcal{L}_{\zeta}^{C C}=\frac{g}{\sqrt{2}} W_{\mu}^{-} \bar{\zeta}_{L} \gamma^{\mu}\left(-\sin \theta^{\prime} P_{L} N_{0}+i \cos \theta \cos \theta^{\prime} P_{L} N_{1}+\sin \theta \cos \theta^{\prime} P_{L} N_{2}\right)+\text { h.c. }
$$


and

$$
\mathcal{L}_{\ell}^{C C}=\frac{g}{\sqrt{2}} W_{\mu}^{-} \bar{\ell}_{L} \gamma^{\mu}\left(\cos \theta^{\prime} P_{L} N_{0}+i \cos \theta \sin \theta^{\prime} P_{L} N_{1}+\sin \theta \sin \theta^{\prime} P_{L} N_{2}\right)+\text { h.c. }
$$

Notice that the neutral current remains flavor diagonal at tree-level [66], hence the heavy neutrinos couple to the SM ones only through the charged current interactions at this order. This is a distinctive feature of our TeV neutrino physics. The SM like Yukawa interactions lead to the following terms involving the Higgs:

$$
\begin{aligned}
& -\frac{m_{D}}{v}\left(\overline{v_{\zeta_{R}}} v_{\zeta_{L}}+\overline{v_{\zeta_{L}}} v_{\zeta_{R}}\right) H=-\frac{m_{D}}{v}\left[\sin \theta \cos \theta \cos \theta^{\prime}\left(\bar{N}_{1} N_{1}+\bar{N}_{2} N_{2}\right)\right. \\
& \left.\quad-i \cos \theta^{\prime}\left(\cos ^{2} \theta-\sin ^{2} \theta\right) \bar{N}_{1} \gamma_{5} N_{2}-i \sin \theta \sin \theta^{\prime} \bar{N}_{1} \gamma^{5} N_{0}-\cos \theta \sin \theta^{\prime} \bar{N}_{2} N_{0}\right] H
\end{aligned}
$$

and

$$
\begin{aligned}
& -\frac{m^{\prime}}{v}\left(\overline{v_{\zeta_{R}}} v_{\ell L}+\overline{v_{\ell L}} v_{\zeta_{R}}\right) H=-\frac{m^{\prime}}{v}\left[\sin \theta \cos \theta \sin \theta^{\prime}\left(\bar{N}_{1} N_{1}+\bar{N}_{2} N_{2}\right)\right. \\
& \left.-i \sin \theta^{\prime}\left(\cos ^{2} \theta-\sin ^{2} \theta\right) \bar{N}_{1} \gamma_{5} N_{2}+i \sin \theta \cos \theta^{\prime} \bar{N}_{1} \gamma^{5} N_{0}+\cos \theta \cos \theta^{\prime} \bar{N}_{2} N_{0}\right] H .
\end{aligned}
$$

The sum of these two expressions is equal to:

$$
-\frac{m_{D}}{v}\left(1+\frac{m^{\prime 2}}{m_{D}^{2}}\right) \cos \theta^{\prime}\left[\sin \theta \cos \theta\left(\bar{N}_{1} N_{1}+\bar{N}_{2} N_{2}\right)-i\left(\cos ^{2} \theta-\sin ^{2} \theta\right) \bar{N}_{1} \gamma_{5} N_{2}\right] H
$$

\section{Heavy Leptons in Minimal Walking Technicolor models}

The simple model presented above is per se inconsistent because of uncanceled gauge and Witten anomalies [3]. To avoid such an inconsistency we add new fermions charged under the electroweak gauge group whose additional new gauge dynamics is responsible for a dynamical breaking of the electroweak symmetry. The model we use as a template is Minimal Walking Technicolor (MWT) [1].

MWT is an $\mathrm{SU}(2)$ technicolor gauge theory with two adjoint technifermion:

$$
Q_{L}^{a}=\left(\begin{array}{l}
U^{a} \\
D^{a}
\end{array}\right)_{L}, \quad U_{R}^{a}, \quad D_{R}^{a}, \quad a=1,2,3,
$$


with $a$ being the adjoint color index of SU(2). The left handed fields are arranged in three doublets of the $\mathrm{SU}(2)_{L}$ weak interactions in the standard fashion. The condensate is $\langle\bar{U} U+\bar{D} D\rangle$ which correctly breaks the electroweak symmetry. Anomalies are canceled by adding a heavy Lepton doublet [4]:

$$
L_{L}=\left(\begin{array}{c}
N \\
E
\end{array}\right)_{L} \quad N_{R}, E_{R} .
$$

In general, the gauge anomalies cancel using the following generic hypercharge assignment

$$
\begin{array}{ll}
Y\left(Q_{L}\right)=\frac{y}{2}, & Y\left(U_{R}, D_{R}\right)=\left(\frac{y+1}{2}, \frac{y-1}{2}\right), \\
Y\left(L_{L}\right)=-3 \frac{y}{2}, & Y\left(N_{R}, E_{R}\right)=\left(\frac{-3 y+1}{2}, \frac{-3 y-1}{2}\right),
\end{array}
$$

where the parameter $y$ can take any real value [4]. One recovers the SM-like hypercharge assignment for $y=1 / 3$. If we choose indeed the SM hypercharge assignment the new lepton family can be identified with the heavy family discussed above with $N=v_{\zeta}$ and

$E=\zeta$. The physics of the strongly coupled sector of MWT has been investigated already in the literature [1, 4, 5, 6] while the focus here is on the heavy leptons, their interplay with the new strong dynamics as well as the mixing with the SM light neutrinos. We note that in addition to the appearance of fundamental heavy leptons canceling the gauge anomalies in the MWT model, both the MWT and the Ultra Minimal Walking Technicolor model feature composite heavy leptons [5, 7]

The low energy effective theory we will use for determining the interesting signals for LHC phenomenology [5, 8] contains composite spin one and spin zero states and we summarize it in the appendix. The new heavy spin one states will mix with the SM gauge bosons and hence modify the charged and flavor currents in Eqs. (16) and Eqs. (17).

\section{PARAMETER SPACE CONSTRAINTS}

There are both direct and indirect constraints from collider experiments as well as cosmological constraints on heavy leptons, which we discuss below. 


\section{A. Limits from LEP and Tevatron}

\section{$\underline{\text { LEP }}$}

For a neutral fourth lepton decaying to SM leptons, mass limits at 95 \% CL from LEP2 were determined in [9] including data up to $\sqrt{s}=208 \mathrm{GeV}$. The study assumed decays of the heavy neutrino into $\ell W$ and assumed that the relevant mixing coefficients square are larger than $10^{-11}$ (such that the heavy neutrino decays inside the detector). The best mass limit was achieved for the $W \mu$ decay mode yielding a mass limit of $101.5 \mathrm{GeV}$ for a Dirac neutrino and $90.7 \mathrm{GeV}$ for a Majorana neutrino, while the $W \tau$ decay mode yields the weakest mass limit of $90.3 \mathrm{GeV}$ for a Dirac neutrino and $80.5 \mathrm{GeV}$ for a Majorana neutrino. The study also assumed a pair production cross-section of the $N_{1}$ corresponding to $\cos \theta=1 \mathrm{in} \mathrm{Eq.} 17$ as also pointed out in [10]. Therefore the lower bound on $M_{1}$ is reduced if $\cos \theta<1$. With no assumption on the size of mixing coefficients an earlier mass limit at 95\% CL from LEP1 for a decaying heavy neutrino was obtained in [11] from the study of the total $\mathrm{Z}$ decay width yielding $44 \mathrm{GeV}$ for a Dirac neutrino and $38.2 \mathrm{GeV}$ for a Majorana neutrino.

In [9] mass limits at $95 \%$ CL were also set on a charged fourth lepton. For a charged decaying fourth lepton, the mass limit achieved is $100.8 \mathrm{GeV}$ for decays into $v W$ and 101.9 $\mathrm{GeV}$ for decays into $N_{1} W$ (assuming the mass splitting between $N_{1}$ and $\zeta$ is at least 15 $\mathrm{GeV})$. For a stable charged lepton the mass limit achieved is $102.6 \mathrm{GeV}$.

For a stable heavy neutrino mass limits arise from the study of the invisible $\mathrm{Z}$ decay width presented below.

\section{Tevatron}

At Tevatron, the CDF collaboration has also searched for single, weakly interacting and long-lived charged particles, or more generally CHAMPs (CHArged Massive Particles). They find an upper limit to the production cross section (times acceptance) of $10 \mathrm{fb}$ at $\sqrt{s}=1.96 \mathrm{TeV}$ at $95 \% \mathrm{CL}$ [12]. For our CHAMP candidate $\zeta^{ \pm}$we are below this bound for masses above $\sim 100 \mathrm{GeV}$.

\section{Invisible width of the $Z$}

From eq. (17), it follows that $M_{1}>M_{Z} / 2$ is a sufficient requirement to forbid any 
contribution from the heavy neutrinos $N_{1,2}$ to the $Z$ decay width. As can be seen from eq.(14), such a requirement implies an upper bound on $m_{R}$ :

$$
m_{R} \leq m_{R \max }=2 \frac{m_{D}^{\prime 2}}{m_{Z}}-\frac{m_{Z}}{2}
$$

As can be also seen from fig, 1, for $m_{R}=0$ this implies $m_{D}^{\prime} \geq M_{Z} / 2$. From eqs.(13) and (25), it turns out that the range of values allowed for $\theta$ is:

$$
\theta_{\min } \equiv \arctan \frac{m_{Z}}{2 m_{D}^{\prime}} \leq \theta \leq 45^{\circ}
$$

The upper bound on $m_{R}$ and the lower bound on $\theta$ are shown respectively in the left and right plots of fig. 2, as a function of $m_{D}^{\prime}$.
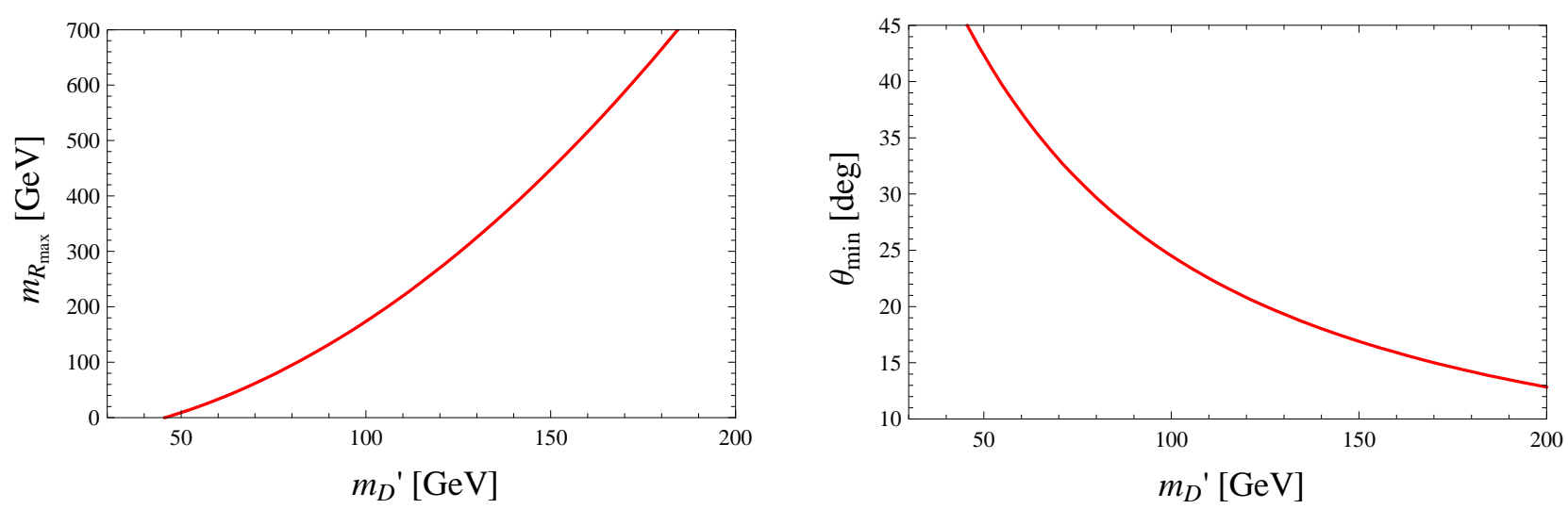

FIG. 2: Left: Upper bound on $m_{R}$ as a function of $m_{D^{\prime}}^{\prime}$ eq. (25). Right: Lower bound on $\theta$ as a function of $m_{D^{\prime}}^{\prime}$ eq. (26).

One could however wonder whether the scenario $M_{1}<M_{Z} / 2<M_{2}$ is still viable, because of the $\cos ^{2} \theta$ suppression in eq.(17). Indeed, by adding the pair of Majorana neutrinos $N_{1}$ and $N_{2}$ to the three light neutrinos of the $\mathrm{SM}$, the effective number of neutrinos involved in the $Z$ invisible width, $\langle N\rangle=\Gamma_{\text {inv }} / \Gamma_{0}$ (where $\Gamma_{0}=\Gamma(Z \rightarrow \bar{v} v)$ is the amplitude for $Z$ to decay into a light, practically massless, neutrino), becomes [13]:

$$
\begin{gathered}
\langle N\rangle=3+\theta\left(M_{Z}-2 M_{1}\right) \cos ^{4} \theta x_{11}+\theta\left(M_{Z}-M_{1}-M_{2}\right) 2 \cos ^{2} \theta \sin ^{2} \theta x_{12} \\
+\theta\left(M_{Z}-2 M_{2}\right) \sin ^{4} \theta x_{22}
\end{gathered}
$$


where $\theta(x)$ is the step function and

$$
x_{i j}=\sqrt{1+\left(\frac{M_{i}}{M_{Z}}\right)^{4}+\left(\frac{M_{j}}{M_{Z}}\right)^{4}-2\left[\left(\frac{M_{i}}{M_{Z}}\right)^{2}+\left(\frac{M_{j}}{M_{Z}}\right)^{2}+\frac{M_{i}^{2} M_{j}^{2}}{M_{Z}^{4}}\right]} \quad, i, j=1,2 .
$$

From the direct measurement of the invisible $Z$ width, we have at present $\langle N\rangle=2.92 \pm 0.05$ [2]. We display in fig. 3 the regions of the $\left(M_{1}, M_{2}\right)$ plane allowed at 3 and $2 \sigma$ (light and dark green respectively). Notice that, at $3 \sigma, M_{1}$ is allowed to be smaller than $M_{Z} / 2$ by less than a couple of $\mathrm{GeV}$.

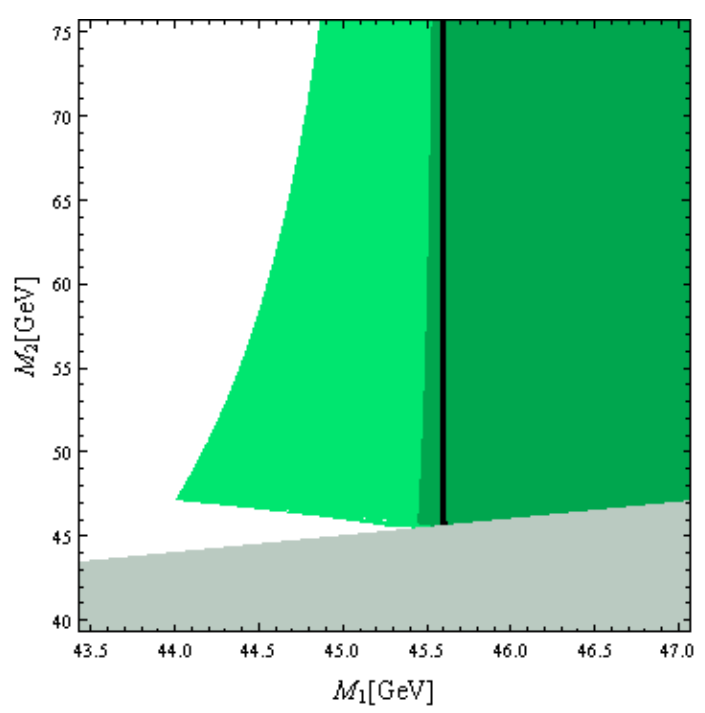

FIG. 3: Allowed region at 3 (light green) and $2 \sigma$ (dark green) in the $\left(M_{1}, M_{2}\right)$ plane. The shaded gray region is excluded by the condition $M_{2}>M_{1}$. The black line corresponds to $\langle N\rangle=3$, namely $M_{1} \leq M_{Z} / 2$.

Actually, even when the $Z$ decays occur below the mass threshold for the production of the heavy states $N_{1,2}$, there could be a deviation from the SM prediction $\langle N\rangle=3$. This is because the standard gauge eigentate $v_{\ell L}$ of eq. (12) is effectively replaced by its normalised projection into the subspace of the light neutrinos, $P_{L} N_{0}$. Since the SM neutrino of flavor $\ell$ mixes with the heavy neutrino $v_{\zeta L}$ belonging to a lepton doublet, the effective number of neutrinos turns out to be slightly increased [14, 15]. At leading order in $\theta^{\prime}$ one has:

$$
\langle N\rangle=3+\frac{3}{2} \sin ^{2} \theta^{\prime},
$$

which applies when the mixing involves $\ell=e, \mu$ (there would be no correction for $\ell=\tau$ ). Given that [2] $\langle N\rangle=2.92 \pm 0.05$, one learns that a possible mixing with the neutrinos of 
flavor $e$ and $\mu$ is subject to the bound $\sin ^{2} \theta^{\prime} \leq 0.047$ at $3 \sigma$. Such a bound is however weaker than the one following from lepton universality, that we now discuss.

\section{B. Direct constraints on mixing}

The mixing angle $\theta^{\prime}$ of the heavy leptons with one SM lepton family (we consider each family in turn) is strongly constrained by lepton universality tests [14, 15]. In addition for the second family there are further constriaints when imposing unitarity of the CKM matrix.

The current status of lepton universality tests is summarised in [16]. The present data verify the universality of the leptonic charged-current couplings to the $0.2 \%$ level. For the mixings with the neutrinos of flavor $e$ and $\mu$, one can extract an upper limit on $\sin ^{2} \theta^{\prime}$ of about 0.012 at $3 \sigma$, while for the mixing with the $\tau$ of about 0.15 at $3 \sigma$.

The CKM unitarity constraint applies only to the mixing with a neutrino of flavor $\mu$ and reads $\sum_{i}\left|V_{u i}\right|^{2}=1 / \cos ^{2} \theta^{\prime}$. Given the present determination [2] $\sum_{i}\left|V_{u i}\right|^{2}=0.9999 \pm 0.0011$, one derives that $\sin ^{2} \theta^{\prime} \leq 0.0033$ at about $3 \sigma$. It should be stressed that the CKM unitarity constraint above can be modified if the charged leptons or the quarks mix with additional exotic particles.

\section{Indirect constraints from EW precision data}

Additional constraints come from Electroweak precision data. The S and $\mathrm{T}$ parameters [17] for a sequential fourth lepton doublet and a right-handed singlet were computed in [18] including both Dirac masses for the doublet and Majorana masses for the righthanded singlet, as in our setup. We give the formulas for arbitrary hypercharge in Appendix C.

The heavy leptons are part of the MWT model, we also consider the contribution from the technicolor sector. Using the one-loop perturbative inspired naive estimate of $S_{\text {naive }}$ we have

$$
S_{\text {naive }}=\frac{1}{6 \pi} d(R) N_{D} \text {, }
$$


where $d(R)=3$ is the dimension of the techni-fermion representation $R$ for MWT while $N_{D}=1$ is the number of electroweak techni-doublets. In a walking Technicolor theory the non-perturbative contributions can futher reduce the $S$ parameter value [19, 20, 21]. In this study we will take $S=0.1$ from the technicolor sector following [5]. In addition to the $S$ and $T$ parameters coming from the new strong dynamics we also have the contribution from the heavy lepton sector. At one loop these two contributions are additive and we will consider from together in the following discussion.

In Fig. 4 we plot the $S$ and T parameters of the MWT model against the experimentally allowed values at the $68 \% \mathrm{CL}$ from [22]. The reference point at which S,T and U vanish was taken to be $M_{H}=150 \mathrm{GeV}$ and $m_{t}=175 \mathrm{GeV}$. The lower ellipsis in Fig. 4 reproduces the one in [22]. The other two ellipses corresponds to simply propagating the first ellipsis to $M_{H}=0.5 \mathrm{TeV}$ and $M_{H}=1 \mathrm{TeV}$, using the $S$ and $\mathrm{T}$ contribution from the heavy Higgs as in [17]. Note that redoing the full fit for the heavy Higgs reference masses results in error ellipses towards more negative $S$ for $M_{H}=0.5,1 \mathrm{TeV}$ in [2]. Also note that the central value for $S$ in [2] is small and negative as opposed to the small and positive central value from the fit in [22] we use here. We tabulate the values of $\frac{M_{1}}{M_{\zeta}}$ for which $S$ and $T$ are within the $68 \%$ CL ellipses (from the upper edge of the top ellipsis to the lower edge of the bottom ellipsis) in table [1.

\begin{tabular}{c||c|c|c} 
& $m_{R}=0$ & $m_{R}=M_{\zeta}$ & $m_{R}=5 M_{\zeta}$ \\
\hline \hline$M_{\zeta}=250 \mathrm{GeV}$ & $0.28-0.72,1.4-1.5$ & $0.12-0.35,1.3-1.33$ & $0.12-0.25$ \\
\hline$M_{\zeta}=500 \mathrm{GeV}$ & $0.65-0.85,1.2-1.25$ & $0.25-0.4,1.17-1.19$ & $0.21-0.25$
\end{tabular}

TABLE I: Table of allowed value of $\frac{M_{1}}{M_{\zeta}}$ within the $68 \mathrm{CL}$ limit for 3 values $m_{R}=M_{2}-M_{1}$ and 2 values of $M_{\zeta}$ with respect to the solid confidence ellipses, corresponding to $M_{H}=150 \mathrm{GeV}$ and $m_{t}=175 \mathrm{GeV}$ [22]

In general the lepton masses are less constrained by the Electroweak precision measurements in the Dirac limit $M_{2}=M_{1}$ and the measurements do not rule out $M_{\zeta}<M_{1}$.

The EW constraints for the MWT model with only a Dirac mass for the 4th lepton were 


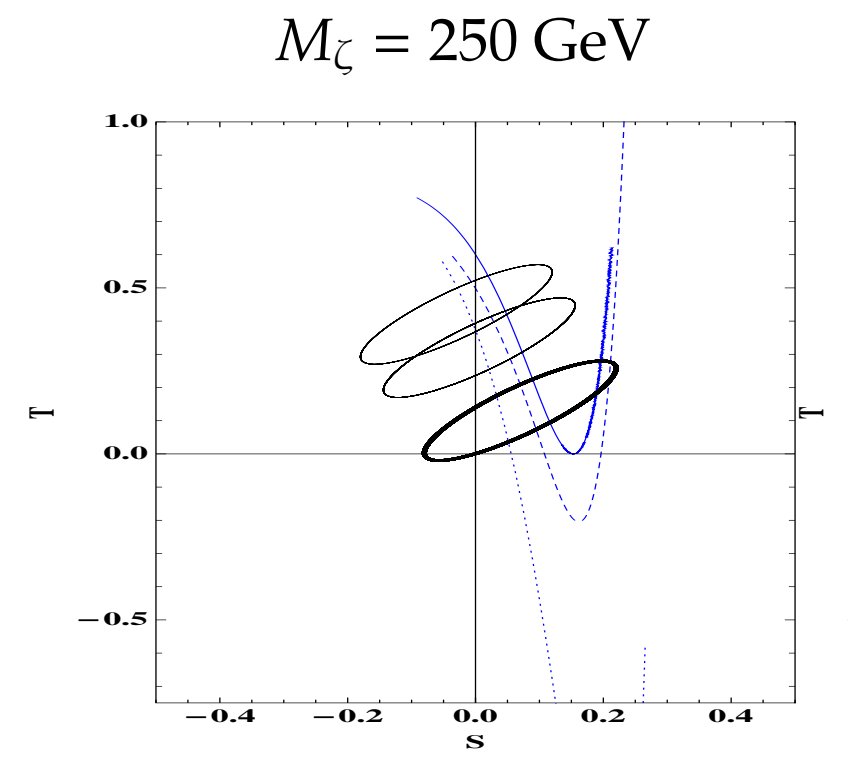

$$
M_{\zeta}=500 \mathrm{GeV}
$$

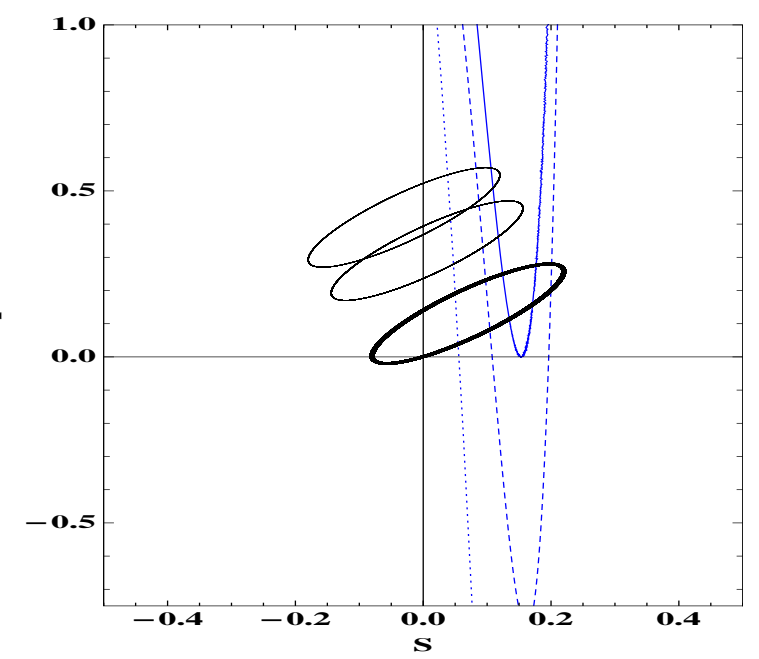

FIG. 4: $S$ and $T$ in the MWT model as a function of $M_{1} / M_{\zeta}$. We assume $S_{M W T}=\frac{1}{2 \pi}, T_{M W T}=0$. $M_{\zeta}=250,500 \mathrm{GeV}$ on the left, right plots respectively and $m_{R} / M_{\zeta}=0,1,5$ for solid, dashed, dotted lines respectively. $M_{1} / M_{\zeta}$ is varied from 0.1 (on the upper left branch) to 1.75 (on the upper right branch) along the hyperbolae. The thick black ellipsis correspond to the $68 \%$ CL confidence level fit given in [22] for $M_{H}=150 \mathrm{GeV}$ and $m_{t}=175 \mathrm{GeV}$. The other two ellipses correspond to $M_{H}=0.5,1 \mathrm{TeV}$ respectively.

considered in detail in [4, 5, 23] while the electroweak parameters in which a Majorana mass is given to the left-handed neutrino were discussed in [24].

\section{Cosmological constraints}

We first consider $M_{\zeta}>M_{1}$. In this regime $\zeta^{ \pm}$decays immediately to $N_{1} W$. Instead $N_{1}$ only decays through mixing with the SM leptons and could in principle be long lived or stable. Assuming the heavy neutrinos are produced as expected in the Big Bang model and that they clump as ordinary baryons, the lower mass limits from CDMS II are 500 $\mathrm{GeV}$ for a Dirac neutrino and no exclusion above $\frac{1}{2} M_{Z}$ for a Majorana neutrino [25]. The lower limit for the Dirac neutrino might be significantly reduced if the neutrinos do not clump like ordinary baryons. If $N_{1}$ is responsible for the dark matter density the mass limits change. This scenario was studied in [26, 27]. It is worth mentioning that MWT models can allow for different type of dark matter candidates [28, 29] of possible interest to recent cosmological observations [30].

Cosmological constraints for $M_{\zeta}<M_{1}$ were considered in [31]. In this regime $\zeta^{ \pm}$ 
will only decay through mixing with the SM leptons and this means $\zeta^{ \pm}$could be long lived, if $\sin \theta^{\prime}$ is sufficiently small in Eq. (16). It is excluded that $\zeta^{ \pm}$could be absolutely stable since this would result in heavy hydrogen, which has not been observed. For a $100-500 \mathrm{GeV}$ charged lepton, the upper limit on the life-time is constrained from big-bang nucleosynthesis [32, 33, 34, 35] to be below $10^{7}-10^{8}$ sec as discussed in [36].

\section{LHC PHENOMENOLOGY}

In this section we investigate aspects of the phenomenology related to the interplay between the new weekly coupled sector, i.e. the heavy leptons with its mixing with the SM fermions, and the new strongly coupled sector breaking the electroweak symmetry dynamically. We consider only the MWT global symmetries relevant for the electroweak sector, i.e. the subsector $S U(2) \times S U(2)$ spontaneously breaking to $S U(2)$. The low energy spectrum contains, besides the composite Higgs, two $S U(2)$ triplets of (axial-) vector spin

one mesons. The effective Lagrangian has been introduced in [5, 37] and summarized in the Appendix $B$ along with the basic input parameters. The spin one massive eigenstates are indicated with $R_{1}$ and $R_{2}$ and are linear combinations of the composite vector/axial mesons of MWT and the weak gauge boson eigenstates. We have implemented the $S U(2) \times S U(2)$ technicolor sector in CalcHEP [38] using the LanHEP module [39] to generate the Feynman rules in [37]. We have added the new leptons to this implementation for the present study.

\section{A. Production and decay of the new leptons}

The heavy leptons may be directly produced through the charged- and neutral current interactions:

$$
\begin{aligned}
& p p \rightarrow W^{ \pm} / R_{1,2}^{ \pm} \rightarrow \zeta^{ \pm} N_{i}, \\
& p p \rightarrow Z / \gamma / R_{1,2}^{0} \rightarrow \zeta^{+} \zeta^{-}, \quad p p \rightarrow Z / R_{1,2}^{0} \rightarrow N_{i} N_{j}, \quad i, j=1,2 .
\end{aligned}
$$

The corresponding Feynman diagrams are given in Fig. 5. The direct production cross sections for the heavy leptons in MWT are largely independent of the parameters asso- 


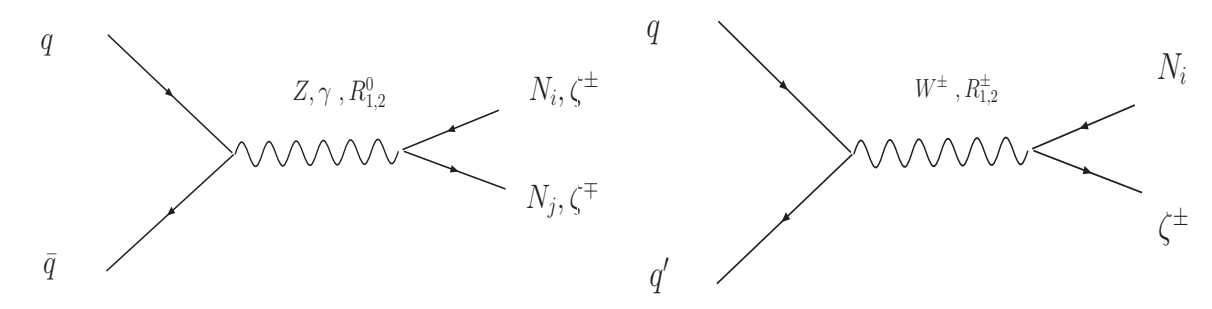

FIG. 5: Feynman diagrams for direct production of the heavy leptons in MWT with $i, j=1,2$.
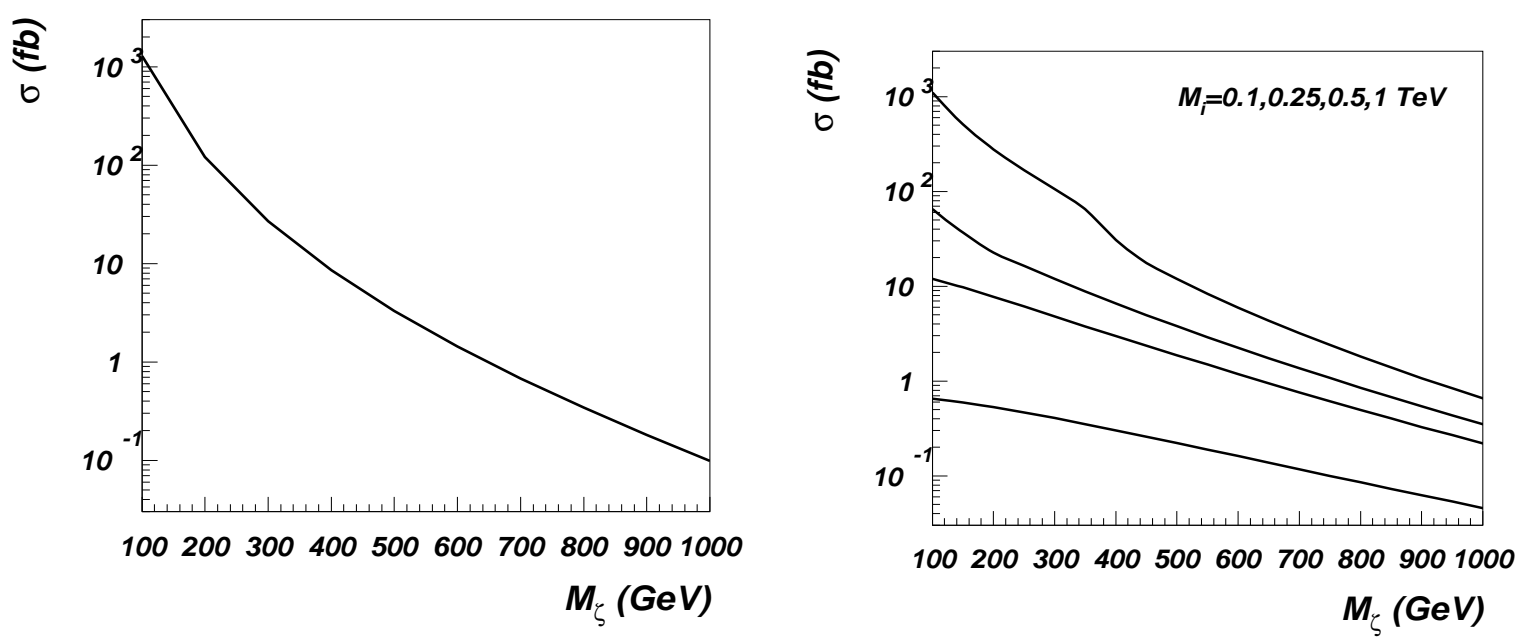

FIG. 6: The LHC production cross section for $p p \rightarrow \zeta^{+} \zeta^{-}$(left) and for $p p \rightarrow \zeta^{+} N_{i}$ for $M_{i}=$ $0.1,0.25,0.5,1 \mathrm{TeV}$ (right), as a function of $M_{\zeta}$. The mixing matrix elements are set to unity.

ciated to the technicolor sector, see Eq. (B5). Hence, in the direct production of $\zeta^{+} \zeta^{-}$the only free parameter is the mass of the charged lepton $M_{\zeta}$. The direct production of $N_{i} N_{j}$ and $\zeta^{ \pm} N_{i}$ depends, in addition to the masses of the leptons, on the $V$ matrix entries of Eq. (12) as follows from Eqs. (16) and (17). We present in Fig. 6 the LHC cross sections for direct pair and single production of $\zeta$ setting the relevant mixing element to one. The actual production cross section is obtained by multiplying the above result by the square of the corresponding mixing element in the matrix $V$. Similarly we present the $N_{i} N_{i}$ direct production cross-section in Fig. 7

The final state distributions arising from the direct production of the leptons depend on the specific parameters of the technicolor sector. In particular $R_{1}$ is a (mostly) axialresonance and $R_{2}$ is a (mostly)vector-resonance, so $R_{1}$ mixes mostly with the $Z$ boson while $R_{2}$ mixes significantly with the photon. Consequently the invariant mass distribution of the heavy neutral leptons $N_{i} N_{i}$ will be relatively more dominated by the $R_{1}$ resonance compared to the charged leptons $\zeta^{+} \zeta^{-}$. This is demonstrated in Fig. 8. The general form 


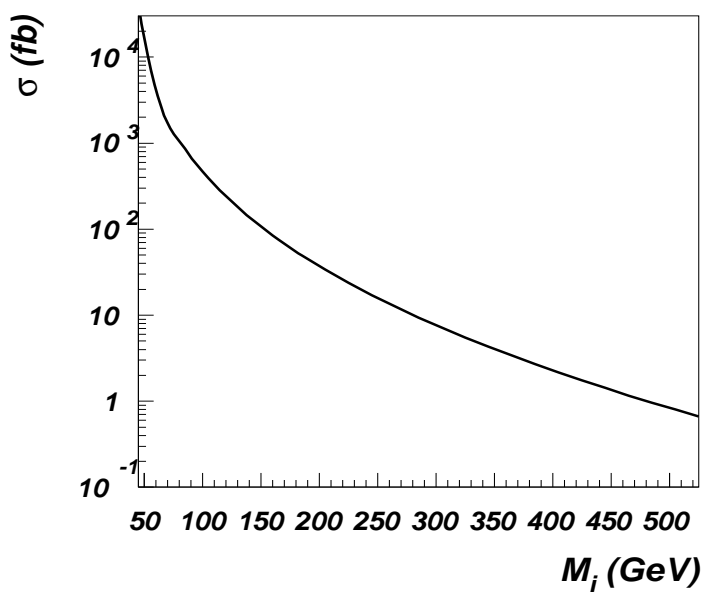

FIG. 7: LHC production cross section for $p p \rightarrow N_{i} N_{i}$ as a function of $M_{i}$ assuming unity mixing matrix element.

of the mixing in the vector sector is given in Eq. (B6). The masses and widths of $R_{1,2}$ as a function of $M_{A}, \tilde{g}$ and $S$ are given for two parameter space points in Eq. (B7) and studied in general in [37].
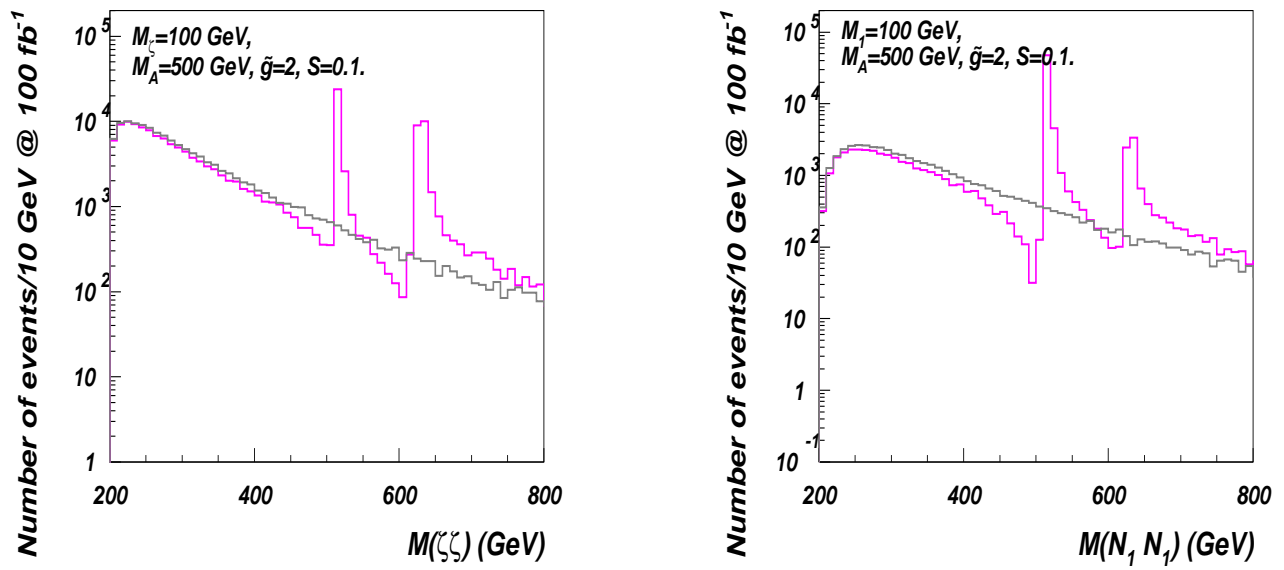

FIG. 8: Invariant mass distributions, $M\left(\zeta^{+} \zeta^{-}\right)$and $M\left(N_{1} N_{1}\right)$ in pair production of $\zeta^{+} \zeta^{-}$(left) and $N_{1} N_{1}$ (right) in the MWT (purple) and when the leptons are added to the SM (grey), assuming unity mixing matrix element. In both frames the first peak corresponds to the $R_{1}$ resonance while the second corresponds to the $R_{2}$ resonance. The neutral current coupling of $N_{1} N_{1}$ is axial and therefore more dominated by the $R_{1}$ resonance. We take the values $\tilde{g}=2, M_{A}=500, S=0.1$ for the parameters of the technicolor sector. The corresponding masses and widths of $R_{1,2}$ are given in Eq. (B7)

Production of the heavy leptons can also proceed via the Yukawa-type couplings to the composite Higgs following from Eq. (20). We are following reference [5] for an effective 
way to give masses to the SM fermions in the MWT setup. The composite Higgs may itself be produced through either gluon fusion, vector boson fusion or in association with a SM vector boson:

$$
g g \rightarrow H, \quad p p \rightarrow q q^{\prime} H, \quad p p \rightarrow H Z / H W .
$$

The process $g g \rightarrow H \rightarrow N_{i} N_{i} \rightarrow W W \mu \mu$ (within the SM framework) was recently considered in [40] where also a 4th generation of quarks were included that enhance the $g g \rightarrow H$ cross section [41], for a recent review of the scenario in which the new leptons are accompanied by a fourth generation of quarks, see [42]. In the MWT class of models this channel is not expected to be enhanced compared to the SM since the techniquarks are not colored.

On the other hand the associate production of the composite Higgs can be enhanced in these technicolor models [37, 43]. In particular the $p p \rightarrow H Z / H W$ channel can be enhanced by the presence of a light axial-vector resonance as shown in [37]. In that study it was also shown that the vector boson fusion production of the composite Higgs is not expected to be enhanced compared to the SM. We will therefore focus, in the collider signature section, on the associate production of the Higgs.

We give expressions for the decay widths of the heavy leptons in Appendix $\mathrm{D}$. The decay patterns depend on the mass hierarchy and the mixings between the leptons. The branching ratio for $N_{1}$ is particularly simple in the regime where $M_{\zeta}>M_{1}$ and $M_{H}>M_{1}$. In this case either $N_{1}$ is absolutely stable, or if it mixes with the SM

$$
\Sigma_{\ell} \operatorname{Br}\left(\mathrm{N}_{1} \rightarrow \ell^{-} \mathrm{W}^{+}\right)=\Sigma_{\ell} \operatorname{Br}\left(\mathrm{N}_{1} \rightarrow \ell^{+} \mathrm{W}^{-}\right)=0.5 .
$$

Assuming Eq. 33 we plot the decay width and lifetime of $N_{1}$ in Fig. 9 . This may be compared with the results in [10]. We note that a value of $\sin \theta^{\prime} \sim 10^{-6}$ would yield a decay length of $\sim 1 \mathrm{~m}$ which is enough for a relativistically boosted particle to escape detection at the LHC and be considered as missing energy in the various processes [44]. 

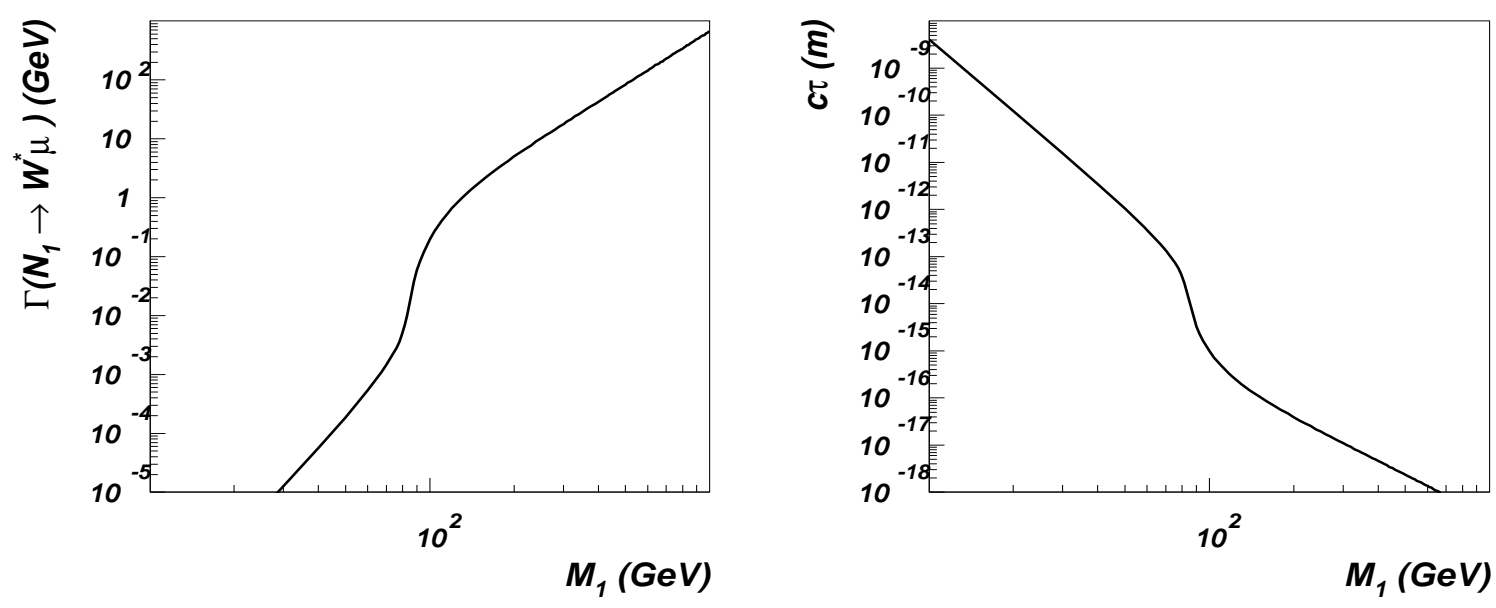

FIG. 9: The width $\Gamma$ (left) and decay length $c \tau$ (right) of $N_{1}$ assuming decays into $W^{*} \mu$ with unity matrix element. To obtain the real width the curve in the left frame should be multiplied by the sum of mixing matrix elements squared while the real decay length is found by dividing the curve in the right frame by the same quantity.

\section{B. Collider signatures of heavy leptons with an exact flavor symmetry}

Let us first consider the limit in which the new leptons do not mix appreciably with the SM ones.

If $M_{\zeta}>M_{1}$ then $N_{1}$ constitutes a long lived neutral particle and will give rise to missing momentum $\not_{T}$ and missing energy $\mathbb{E}_{T}$ signals. In particular the decay mode $H \rightarrow N_{1} N_{1}$ gives rise to an invisible partial width of the composite Higgs.

As pointed out in [37, 43], the cross-section for $\mathrm{ZH}$ production can be enhanced in MWT models because the axial-vector resonance can be light [5, 20]. Here the $\ell^{+} \ell^{-}+\not_{T}$ state will receive contributions both from $Z H$ and $N_{1}, N_{2}$ production, as shown in Fig. 10 We therefore study the proces $p p \rightarrow Z N_{1} N_{1} \rightarrow \ell^{+} \ell^{-}+p_{T}$. We consider limiting values

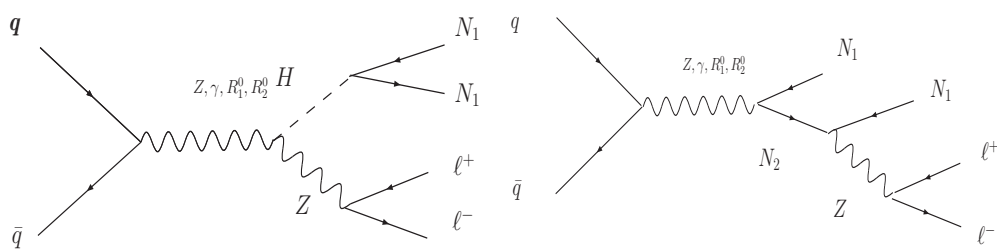

FIG. 10: Feynman diagrams for the $\ell^{+} \ell^{-}+\not \phi_{T}$ signal due to heavy leptons in the MWT model

of the parameters such that either the Higgs or the $N_{2}$ state is too heavy to contribute significantly as well as parameters where both contribute in the process. 
The acceptance cuts relevant for LHC are

$$
\left|\eta^{\ell}\right|<2.5, \quad p_{T}^{\ell}>10 \mathrm{GeV}, \quad \Delta R(\ell \ell)>0.4
$$

Here $\ell$ is a charged lepton, $\eta^{\ell}$ and $p_{T}^{\ell}$ are the pseudo-rapidity and transverse momentum of a single lepton while $\Delta R$ measures the separation between two leptons in the detector. $\Delta R$ is defined via the difference in azimuthal angle $\Delta \phi$ and rapidity $\Delta \eta$ between two leptons as $\Delta R \equiv \sqrt{(\Delta \eta)^{2}+(\Delta \phi)^{2}}$.

The main sources of background come from di-boson production followed by leptonic decays [47, 49, 50]

$$
\mathrm{ZZ} \rightarrow \ell^{+} \ell^{-} v \bar{v}, W^{+} W^{-} \rightarrow \ell^{+} v \ell^{-} \bar{v}, \mathrm{ZW} \rightarrow \ell^{+} \ell^{-} \ell v
$$

where in the last process the lepton from the $\mathrm{W}$ decay is missed.

We impose the additional cuts

$$
\left|M_{\ell \ell}-M_{Z}\right|<10 \mathrm{GeV}, \text { and } \Delta \phi(\ell \ell)<2.5
$$

The first is meant to reduce the WW background by requiring the invariant mass of the lepton pair to reproduce the $\mathrm{Z}$ boson mass. The second cut on the azimuthal angle separation together with taking large $\phi_{T}$ reduces potential backgrounds such as single $Z$ production + jets with fake $h_{T}[49,50]$.

The results are given in Fig. 11 assuming a fully invisibly decaying Higgs. On the left panel we show the signal and background arising from the SM featuring the new heavy leptons. On the right hand panel we show the same signal but in the MWT model. For $\phi_{T}>100 \mathrm{GeV}$ where the signal could potentially be observed, the Higgs production channel dominates and in the MWT model a very distinct $\eta_{T}$ distribution arises due to the effect of the $R_{1}$ resonance. While invisible decays of a SM model Higgs at most appear as an excess of events compared to the background in e.g. $\not_{T}$ distributions, the presence of a light axial-vector resonance results in a peaked distribution, different from the shape of the background, making it a more striking signal. This was also found in the context of Higgs decays to technibaryon Dark Matter candidates in [46]. The peak will degrade for smaller values of $\tilde{g}$. However, for a light axial resonance a relatively large value of $\tilde{g}$ is 
favored by unitarity arguments [61, 62] and electroweak precision observables [5, 37, 63] The peak will also degrade and move to higher $\phi_{T}$ for larger mass of the axial resonance. Also, in Fig. 11 we have assumed a fully invisibly decaying Higgs. The true invisible
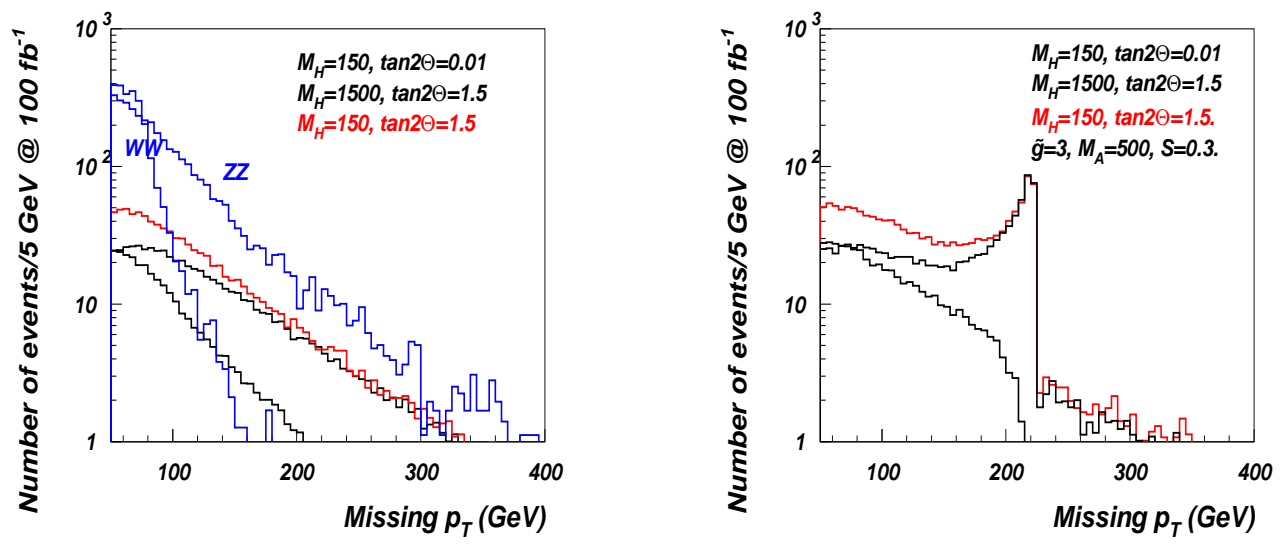

FIG. 11: $\ell^{+} \ell^{-}+p_{T}$ signal from $p p \rightarrow Z N_{1} N_{1} \rightarrow \ell^{+} \ell^{-} N_{1} N_{1}$ in the SM (left) and in the MWT (right). In blue the SM background from ZZ and WW production. Upper black line corresponds to $M_{H}=150$ $\mathrm{GeV}, M_{1}=50 \mathrm{GeV}, \tan 2 \theta=0.01\left(M_{2}=20 \mathrm{TeV}\right)$; Lower black line corresponds to $M_{H}=1500 \mathrm{GeV}$, $M_{1}=50 \mathrm{GeV}, \tan 2 \theta=1.5\left(M_{2}=175 \mathrm{GeV}\right)$; Red line corresponds to $M_{H}=150 \mathrm{GeV}, M_{1}=50 \mathrm{GeV}$, $\tan 2 \theta=1.5\left(M_{2}=175 \mathrm{GeV}\right)$

branching fraction of the Higgs will depend on the mass of the $N_{1}$ particle as shown in Fig. 12 .

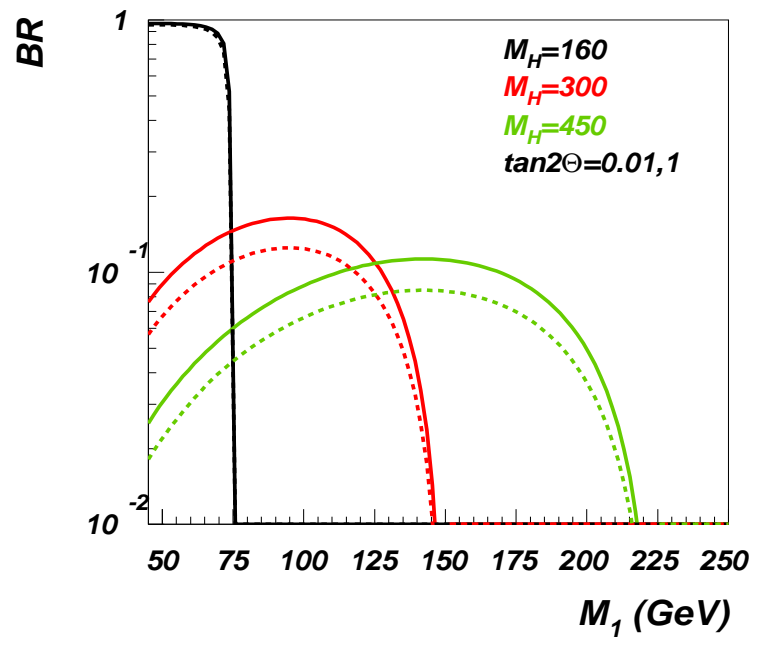

FIG. 12: The invisible branching fraction of the composite Higgs $\mathrm{Br}\left[\mathrm{H} \rightarrow \mathrm{N}_{1} \mathrm{~N}_{1}\right]$

An invisible partial width of the composite Higgs has been searched for at LEP in the proces $e^{+} e^{-} \rightarrow H Z$ with $\mathrm{Z}$ decaying hadronically [45]. However, no limits were achieved for $M_{H}>114 \mathrm{GeV}$. The same final state from $H Z / H W$ production in $p p$ collisions has been 
considered by several authors, also recently in the context of technicolor models [46]. See [46] for references to the literature. The LHC discovery potential for an invisibly decaying Higgs in this final state at LHC has also been investigated at detector level [47, 48]. It was found that the $H W$ mode is not promising [48] while the $\mathrm{ZH}$ mode remains challenging. With a SM production cross section of $H Z$ a significance of $3.43 \sigma$ was achieved at $M_{H}=160$ Gev dropping to a $2 \sigma$ excess at $M_{H}=200 \mathrm{GeV}$ [47].

Thanks to the possible increase in the $H Z$ production cross section found in [37] together with the resonance like structure in the $\phi_{T}$ distribution displayed in Fig. 11, we believe that this channel could be interesting to investigate the interplay between new long-lived heavy neutrinos and composite vector states at LHC.

If instead $M_{\zeta}<M_{1}$ then $\zeta$ can be a long-lived CHAMP (Charged Massive Particle). Collider signatures of long lived charged leptons could either be displaced vertices or, if the charged lepton decays outside the detector, a muon like signal for which the heavy mass should be reconstructable. Such a long-lived CHAMP arises in several scenarios and has been study in some detail, for a review see e.g. [51].

In [52] a Herwig based study of the LHC reach for long-lived leptonic CHAMPs was considered, based on a direct production cross-section identical to that in Fig. 6. With a discovery criterion of 5 pairs of reconstructed opposite charge heavy leptons a reach of $M_{\zeta}=950 \mathrm{GeV}$ at $100 \mathrm{fb}^{-1}$ was found, reduced to $800 \mathrm{GeV}$ without specialized triggers. We can expect this reach to be improved in our model by searching also for single $\zeta$ production channel in Fig. 6. Additionally it was found that long-lived leptonic and scalar CHAMPS could be dstinguished for masses up to $580 \mathrm{GeV}$.

The discovery potential for long-lived CHAMPS has also been studied at detector level for LHC. The CMS and Atlas collaboration has considered various long-lived CHAMPS [53]. From their results we infer that 3 signal events with less than one background event could be observed in CMS with $1 \mathrm{fb}^{-1}$ and $M_{\zeta} \sim 300 \mathrm{GeV}$ and similar in Atlas. More precisely in [53] 3 signal events could be seen in direct pair production of $300 \mathrm{GeV} \mathrm{KK}$ taus with a pair production cross-section of $20 \mathrm{fb}$, very similar to what we find in Fig. 6 Figure 8 shows that it is interesting to investigate the invariant mass distribution of the leptonic CHAMP. 


\section{Collider Signatures of Promiscuous Heavy Leptons}

If the heavy leptons mix with the SM leptons, this will give rise to Lepton Number Violating (LNV) processes with same sign leptons and jets in the final state, e.g.

$$
\begin{aligned}
& p p \rightarrow W^{ \pm} / R_{1,2}^{ \pm} \rightarrow \mu^{ \pm} N_{i} \rightarrow \mu^{ \pm} \mu^{ \pm} W^{\mp} \rightarrow \mu^{ \pm} \mu^{ \pm} j j \\
& p p \rightarrow Z / R_{1,2}^{0} \rightarrow N_{i} N_{j} \rightarrow \mu^{ \pm} \mu^{ \pm} W^{\mp} W^{\mp} \rightarrow \mu^{ \pm} \mu^{ \pm} j j j j, \quad i=1,2,
\end{aligned}
$$

as in Fig. 13 .

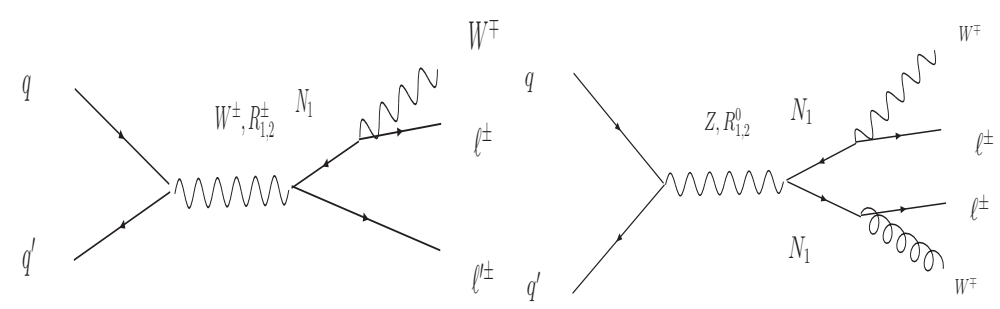

FIG. 13: Same sign leptons from production of $N_{i}$. We will consider the case where the W's decay to jets. Such that the final states we consider are $\mu^{ \pm} \mu^{ \pm} j j$ (left) and $\mu^{ \pm} \mu^{ \pm} j j j j$ (right)

The production cross section $p p \rightarrow N_{1} N_{1} \rightarrow \mu^{ \pm} \mu^{ \pm} j j j j$ may be inferred from Fig. 7 using the branching in Eq. (33) (assuming $M_{\zeta}>M_{1}$ and $M_{H}>M_{1}$ ). The production cross-section for $p p \rightarrow \mu^{ \pm} \mu^{ \pm} j$ is given in in Fig. 14. To compare with [54] we have taken $\cos \theta \sin \theta^{\prime}=0.098$ and $\tan 2 \theta=0$ such that $N_{2}$ is decoupled. More generally the contribution from $\mathrm{N}_{2}$ will be supressed both by the heavier mass and the smaller mixing matrix element.

The potential for observing the $\mu^{ \pm} \mu^{ \pm} j j$ final state has been extensively studied in scenarios with heavy right-handed neutrino singlets, both in the 3v-SM [10, 55, 56, 57] and in the presence of additional new physics [44, 58]. Same sign lepton final states have been searched for at the Tevatron in [59, 60]. The $p p \rightarrow W^{ \pm} \rightarrow \ell^{ \pm} N_{i} \rightarrow \ell^{ \pm} \ell^{ \pm} j j$ process in the 3v-SM was studied in [54] at the level of a fast detector simulation. While backgrounds for same sign lepton production are smaller than for opposite sign lepton production, arising in the Dirac limit, they were found to be significantly larger than previously estimated in parton level processes, in particular for $M_{i}<M_{W}$.

Again the production cross-sections are largely unaffected by the presence of heavy vectors and is as shown in Fig. 14 . However the shape of the distributions are affected by 


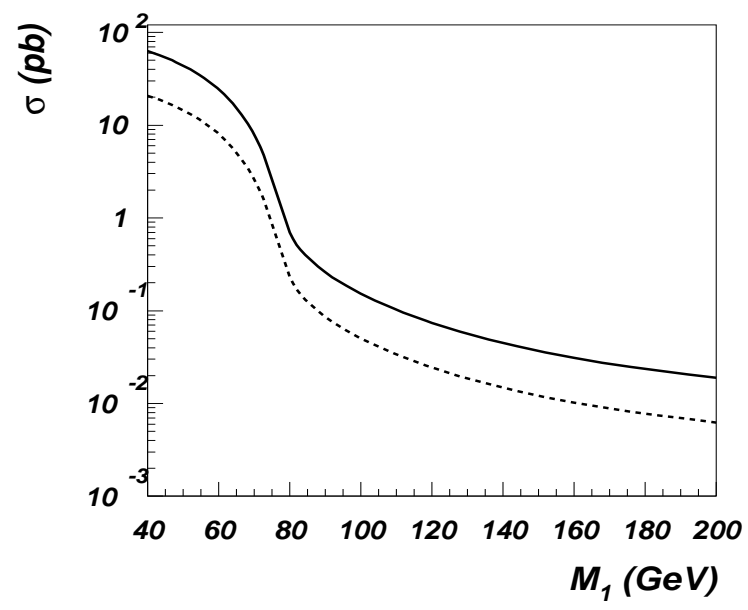

FIG. 14: LHC cross-sections: $\sigma\left(p p \rightarrow N_{1} \mu^{ \pm}\right)$(solid black) and $\sigma\left(p p \rightarrow \mu^{ \pm} \mu^{ \pm} j j\right)$ (dotted black) without imposing any cuts. We take $\sin \theta^{\prime}=0.098$ and $\cos \theta=1$

the presence of the heavy vectors.

To study these processes we impose jet acceptance cuts in addition to the leptonic acceptance cuts given in Eq. (34)

$$
\left|\eta^{j}\right|<3, \quad p_{T}^{j}>20 \mathrm{GeV}, \quad \Delta R(\ell j)>0.5 .
$$

The resulting invariant mass distributions for $\mu^{-} \mu^{-} j j$ (left) and $\mu^{-} \mu^{-} j j j j$ (right) are given below in Fig. 15. We have again taken $\cos \theta=1, \sin \theta^{\prime}=0.098$. While $\sin \theta^{\prime}$ determines the mixing between $N_{1}$ and $\ell$ and therefore is constrained by experiment, $\cos \theta$ is not. This means that the production cross section of $N_{1}, N_{1}$ potentially is significantly larger than the $N_{1} \mu$ production cross-section, compare Figs. 7(left) and 14. If at the same time $N_{1}$ only decays to $W \mu$ as in Eq. (33) with $\operatorname{Br}\left(N_{1} \rightarrow W^{+} \mu^{-}\right)=\frac{1}{2}$ as the only mode we find the result given in the right frame of Fig. 15

The above shows that the interplay of heavy neutrinos and composite vector mesons can lead to striking signatures at the LHC.

\section{AN INTRIGUING STRUCTURE}

We argued that linking the 4th lepton family to a technicolor sector rather than to a fourth family of ordinary quarks renders its presence at the electroweak scale natural. 

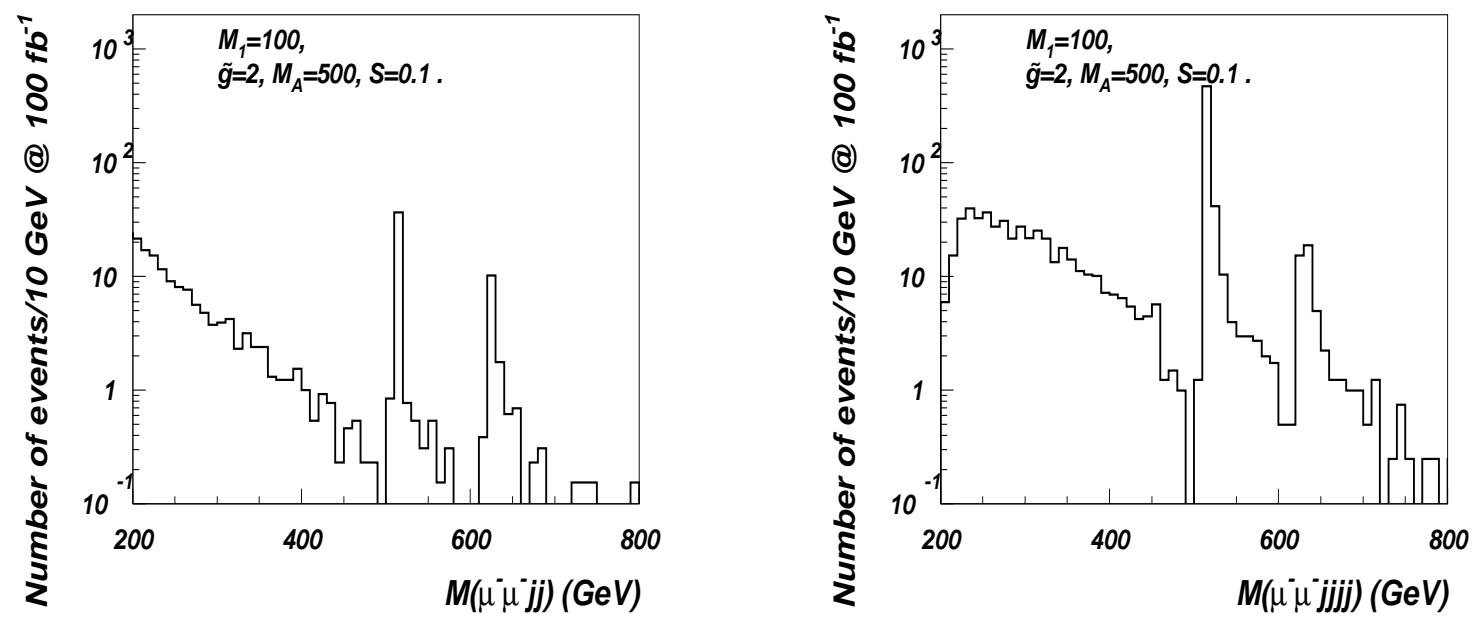

FIG. 15: Invariant mass distributions: $p p \rightarrow N_{1} \mu^{-} \rightarrow \mu^{-} \mu^{-} j j$ (left) and $p p \rightarrow N_{1} N_{1} \rightarrow \mu^{-} \mu^{-} j j j j$ (right). The parameters in the technicolor sector are fixed to be $M_{A}=500 \mathrm{GeV}, S=0.1, \tilde{g}=2$ while the new lepton sector parameters are $M_{1}=100 \mathrm{GeV}, \cos \theta=1, \sin \theta^{\prime}=0.098 . N_{2}$ is decoupled for these parameters.

What is even more interesting is that this link makes the new leptons much less conventional than a sequential type fourth generation. If a grand unification mechanism is at play it is natural to expect that at some energy scale (higher than the weak scale) the weak group extends to [64]:

$$
S U_{1}(2) \times U_{1}(1) \times S U_{2}(2) \times U_{2}(1)
$$

The SM leptons and quarks are uncharged under the first copy of $S U(2) \times U(1)$ while they have the standard charges under the second copy. The fourth family of leptons has the SM like assignment under the first copy but is uncharged under the second copy. We reproduce the table of charges from [64] below: This assignment allows to arrange the low energy matter fields into complete representations of $S U(5) \times S U(5)$. We summarize in table [II] the technicolor and SM fermions transformation properties with respect to the grand unified group. Here the fields $A$ and $F$ are standard Weyl fermions and the gauge couplings of the two $S U(5)$ groups need to be the same. This is a minimal embedding and others can be envisioned.

We further require the extended gauge group to spontaneously break to a single $S U(2) \times$ $U(1)$ which we then identify with the SM weak and hypercharge gauge group. We do not speculate on the mechanism behind the breaking of the extended group and immediately introduce a two by two complex field $\widetilde{H}$ transforming with respect to the extended group 
TABLE II: MWT + One SM Family enlarged gauge group

\begin{tabular}{c|c|c|c|c|c|c} 
& $S O_{T C}(3)$ & $S U_{1}(2)$ & $U_{1}(1)$ & $S U_{c}(3)$ & $S U_{2}(2)$ & $U_{2}(1)$ \\
\hline \hline$q_{L}$ & 1 & 1 & 0 & 3 & 2 & $1 / 6$ \\
$u_{R}$ & 1 & 1 & 0 & 3 & 1 & $2 / 3$ \\
$d_{R}$ & 1 & 1 & 0 & 3 & 1 & $-1 / 3$ \\
$L$ & 1 & 1 & 0 & 1 & 2 & $-1 / 2$ \\
$e_{R}$ & 1 & 1 & 0 & 1 & 1 & -1 \\
\hline$Q_{L}$ & 3 & 2 & $1 / 6$ & 1 & 1 & 0 \\
$U_{R}$ & 3 & 1 & $2 / 3$ & 1 & 1 & 0 \\
$D_{R}$ & 3 & 1 & $-1 / 3$ & 1 & 1 & 0 \\
$\mathcal{L}_{L}$ & 1 & 2 & $-1 / 2$ & 1 & 1 & 0 \\
$\zeta_{R}$ & 1 & 1 & -1 & 1 & 1 & 0
\end{tabular}

TABLE III: GUT

\begin{tabular}{c|c|c} 
& $S U(5)$ & $S U(5)$ \\
\hline \hline $\bar{A}_{S M}$ & 1 & $\overline{10}$ \\
$F_{S M}$ & 1 & 5 \\
\hline $\bar{A}_{M W T}$ & $\overline{10}$ & 1 \\
$F_{M W T}$ & 5 & 1
\end{tabular}

as follows:

$$
\widetilde{H} \quad(2,1 / 2,2,-1 / 2) .
$$

The covariant derivative is:

$$
D \widetilde{H}=\partial \widetilde{H}-i g_{1} W_{1} \widetilde{H}-i \frac{1}{2} g_{1}^{\prime} B_{1} \widetilde{H}+i g_{2} \widetilde{H} W_{2}+i \frac{1}{2} g_{2}^{\prime} B_{2} \widetilde{H}
$$

where we have suppressed the Lorentz indices and

$$
W_{1 / 2}=W_{1 / 2}^{a} \frac{\sigma^{a}}{2}
$$

are the intermediate massless gauge bosons for the two non abelian SU(2) groups while the $B$ s are the gauge bosons for the abelian part. $\sigma^{a}$ are the Pauli matrices normalized according to $\operatorname{Tr}\left[\sigma^{a} \sigma^{b}\right]=2 \delta^{a b}$. 
When this scalar field acquires a diagonal nonzero vacuum expectation value

$$
<\widetilde{H}>=\tilde{v} \mathbf{1}
$$

then the kinetic term for $\widetilde{H}$ evaluated on the vacuum reads:

$$
\operatorname{Tr}\left[D \widetilde{H}^{\dagger} D \widetilde{H}\right] \rightarrow \frac{\tilde{v}^{2}}{2}\left[\left(g_{1}^{\prime} B_{1}-g_{2}^{\prime} B_{2}\right)^{2}+\sum_{a=1}^{3}\left(g_{1} W_{1}^{a}-g_{2} W_{2}^{a}\right)^{2}\right] .
$$

We identify four massive states :

$$
B^{\prime}=\cos \beta B_{1}-\sin \beta B_{2}, \quad W^{\prime a}=\cos \omega W_{1}^{a}-\sin \omega W_{2}^{a}
$$

with mass

$$
M_{B^{\prime}}=\tilde{v} \sqrt{g_{1}^{\prime 2}+g_{2}^{\prime 2}}, \quad M_{W^{\prime}}=\tilde{v} \sqrt{g_{1}^{2}+g_{2}^{2}}
$$

and four massless ones:

$$
B=\cos \beta B_{2}+\sin \beta B_{1}, \quad W^{a}=\cos \omega W_{2}^{a}+\sin \omega W_{1}^{a},
$$

which we identify with the SM states. The two mixing angles are related to the gauge couplings as follow

$$
\cos \beta=\frac{g_{1}^{\prime}}{\sqrt{g_{1}^{\prime 2}+g_{2}^{\prime 2}}} \quad \sin \beta=\frac{g_{2}^{\prime}}{\sqrt{g_{1}^{\prime 2}+g_{2}^{\prime 2}}}
$$

and

$$
\cos \omega=\frac{g_{1}}{\sqrt{g_{1}^{2}+g_{2}^{2}}} \quad \sin \omega=\frac{g_{2}}{\sqrt{g_{1}^{2}+g_{2}^{2}}}
$$

The covariant derivative acting on purely SM fields before breaking the extended symmetry is:

$$
D=\partial-i g_{2} W_{2}^{a} T^{a}-i g_{2}^{\prime} Y B_{2}, \quad \mathrm{SM}-\text { particles }
$$


with $T^{a}$ the $S U_{2}(2)$ generators and $Y$ the generator of $U_{2}(1)$ for a generic SM particle. After symmetry breaking the covariant derivative becomes:

$$
D=\partial-i g W^{a} T^{a}-i g^{\prime} Y B+i g \tan \omega W^{\prime a} T^{a}+i g^{\prime} \tan \beta Y B^{\prime}, \quad \mathrm{SM}-\text { particles }
$$

with $g=g_{2} \cos \omega$ and $g^{\prime}=g_{2}^{\prime} \cos \beta$ the SM couplings.

For the new leptons and techniquarks $(Q)$ the electroweak covariant derivative, after symmetry breaking, and in terms of the gauge bosons mass eigenstates is:

$$
D=\partial-i g W^{a} T^{a}-i g^{\prime} Y B-i \frac{g}{\tan \omega} W^{\prime a} T^{a}-i \frac{g^{\prime}}{\tan \beta} Y B^{\prime}, \quad \text { New }- \text { Leptons \& } Q
$$

This shows that the new leptons have the same coupling of the SM leptons to the SM gauge fields but will couple differently to the new gauge bosons. In the limit in which $\tilde{v}$ is much larger than the electroweak symmetry we recover the previous model analysis. The constraints and phenomenological consequences of the model we have just introduced will be investigated elsewhere.

\section{CONCLUSIONS AND OUTLOOK}

We have investigated the LHC phenomenology of a fourth family of leptons whose appearance is justified by the presence of a new strongly coupled sector. The latter is responsible for the breaking of the electroweak symmetry dynamically. We have chosen as template for our analysis MWT. In this way the fourth family as well as the Higgs sector of the SM are natural theories. We analyzed a general heavy neutrino mass structure with

and without mixing with the SM families. We have then shown that the interplay of heavy neutrinos and composite vector mesons can lead to striking signatures at the LHC. Finally we introduced a model uniting the fourth lepton family and the technifermion sector at higher energies.

\section{Note added}

While this work was being completed the paper [65] appeared in which some of the ideas considered here were also discussed. 


\section{APPENDIX A: FULL MIXING}

The mixing between the neutrinos of flavor $\zeta$ and the $3 \mathrm{SM}$ neutrinos is described by the $5 \times 5$ mass matrix $\mathcal{M}$ :

$$
-\mathcal{L}=\frac{1}{2}\left(\overline{v_{e L}} \overline{v_{\mu L}} \overline{v_{\tau L}} \overline{v_{\zeta L}} \overline{\left(v_{\zeta R}\right)^{c}}\right)\left(\begin{array}{ccccc}
O(e V) & O(e V) & O(e V) & O(e V) & m_{e}^{\prime} \\
O(e V) & O(e V) & O(e V) & O(e V) & m_{\mu}^{\prime} \\
O(e V) & O(e V) & O(e V) & O(e V) & m_{\tau}^{\prime} \\
O(e V) & O(e V) & O(e V) & O(e V) & m_{D} \\
m_{e}^{\prime} & m_{\mu}^{\prime} & m_{\tau}^{\prime} & m_{D} & m_{R}
\end{array}\right)\left(\begin{array}{c}
\left(v_{e L}\right)^{c} \\
\left(v_{\mu L}\right)^{c} \\
\left(v_{\tau L}\right)^{c} \\
\left(v_{\zeta L}\right)^{c} \\
v_{\zeta R}
\end{array}\right)+\text { h.c. . }
$$

Barring unnatural tunings and up to corrections to its mixings of $O\left(e V / M_{1,2}\right)$, the unitary matrix $V$

$$
\left(\begin{array}{c}
v_{e L} \\
v_{\mu L} \\
v_{\tau L} \\
v_{\zeta L} \\
\left(v_{\zeta R}\right)^{c}
\end{array}\right)=V\left(\begin{array}{c}
P_{L} N_{e} \\
P_{L} N_{\mu} \\
P_{L} N_{\tau} \\
P_{L} N_{1} \\
P_{L} N_{2}
\end{array}\right), V=\left(\begin{array}{ccccc}
c_{e} & -s_{e} s_{\mu} & -s_{e} c_{\mu} s_{\tau} & s_{e} c_{\mu} c_{\tau} & 0 \\
0 & c_{\mu} & -s_{\mu} s_{\tau} & -s_{\mu} c_{\tau} & 0 \\
0 & 0 & c_{\tau} & -s_{\tau} & 0 \\
i c s_{e} & i c_{e} s_{\mu} & i c c_{e} c_{\mu} s_{\tau} & i c_{e} c_{\mu} c_{\tau} & -i s \\
s s_{e} & s c_{e} s_{\mu} & s c_{e} c_{\mu} s_{\tau} & s c_{e} c_{\mu} c_{\tau} & c
\end{array}\right),
$$

where

$$
\begin{aligned}
& t_{\tau}^{2}=\frac{m_{\tau}^{\prime 2}}{m_{D}^{2}}, \quad t_{\mu}^{2}=\frac{m_{\mu}^{\prime 2}}{m_{D}^{2}+m_{\tau}^{\prime 2}} \quad, \quad t_{e}^{2}=\frac{m_{e}^{\prime 2}}{m_{D}^{2}+m_{\tau}^{\prime 2}+m_{\mu}^{\prime 2}} \\
& \tan (2 \theta)=2 \frac{m_{D}^{\prime}}{m_{R}}, \quad m_{D}^{\prime 2}=m_{D}^{2}+m_{\tau}^{\prime 2}+m_{\mu}^{\prime 2}+m_{e}^{\prime 2},
\end{aligned}
$$

diagonalises the lower sector of the $5 \times 5$ mass matrix $\mathcal{M}$, namely

$$
V \mathcal{M} V^{+}=\left(\begin{array}{ccc}
m_{3 \times 3}^{e f f} & 0 & 0 \\
0 & M_{1} & 0 \\
0 & 0 & M_{2}
\end{array}\right)
$$


where the elements of $m_{3 \times 3}^{e f f}$ are naturlly $O(e V)$ and

$$
M_{1}=\frac{m_{R}}{2}\left(\sqrt{1+4 \frac{m_{D}^{\prime 2}}{m_{R}^{2}}}-1\right), \quad M_{2}=\frac{m_{R}}{2}\left(\sqrt{1+4 \frac{m_{D}^{\prime 2}}{m_{R}^{2}}}+1\right) .
$$

At this stage, the matrix in eq. (A4) can be fully diagonalised with a further unitary matrix, which can be identified with the MNS mixing matrix, acting only on the upper $3 \times 3$ block:

$$
U^{+} m_{3 \times 3}^{e f f} U^{*}=\operatorname{diag}\left(m_{1}, m_{2}, m_{3}\right)
$$

\section{APPENDIX B: MWT EFFECTIVE LAGRANGIAN}

Following Ref. [5] and [8] the MWT effective Lagrangian containing spin one and spin zero states reads:

$$
\begin{aligned}
\mathcal{L}_{\text {boson }} & =-\frac{1}{2} \operatorname{Tr}\left[\widetilde{W}_{\mu \nu} \widetilde{W}^{\mu v}\right]-\frac{1}{4} \widetilde{B}_{\mu v} \widetilde{B}^{\mu v}-\frac{1}{2} \operatorname{Tr}\left[F_{\mathrm{L} \mu v} F_{\mathrm{L}}^{\mu v}+F_{\mathrm{R} \mu v} F_{\mathrm{R}}^{\mu v}\right] \\
& +m^{2} \operatorname{Tr}\left[C_{\mathrm{L} \mu}^{2}+C_{\mathrm{R} \mu}^{2}\right]+\frac{1}{2} \operatorname{Tr}\left[D_{\mu} M D^{\mu} M^{\dagger}\right]-\tilde{g}^{2} r_{2} \operatorname{Tr}\left[C_{\mathrm{L} \mu} M C_{\mathrm{R}}^{\mu} M^{\dagger}\right] \\
& -\frac{i \tilde{g} r_{3}}{4} \operatorname{Tr}\left[C_{\mathrm{L} \mu}\left(M D^{\mu} M^{\dagger}-D^{\mu} M M^{\dagger}\right)+C_{\mathrm{R} \mu}\left(M^{\dagger} D^{\mu} M-D^{\mu} M^{\dagger} M\right)\right] \\
& +\frac{\tilde{g}^{2} S}{4} \operatorname{Tr}\left[C_{\mathrm{L} \mu}^{2}+C_{\mathrm{R} \mu}^{2}\right] \operatorname{Tr}\left[M M^{\dagger}\right]+\frac{\mu^{2}}{2} \operatorname{Tr}\left[M M^{\dagger}\right]-\frac{\lambda}{4} \operatorname{Tr}\left[M M^{\dagger}\right]^{2}
\end{aligned}
$$

where $\widetilde{W}_{\mu v}$ and $\widetilde{B}_{\mu v}$ are the ordinary electroweak field strength tensors, $F_{\mathrm{L} / \mathrm{R} \mu v}$ are the field strength tensors associated to the vector meson fields $A_{\mathrm{L} / \mathrm{R} \mu} . V=\frac{1}{\sqrt{2}}\left(A_{L}+A_{R}\right), A=$ $\frac{1}{\sqrt{2}}\left(A_{L}-A_{R}\right)$ are the mass eigenstates in the $g, g^{\prime} \rightarrow 0$ limit (the analogs of the QCD $\rho$ and $a_{0}$ vector and axial-vector mesons). The $C_{\mathrm{L} \mu}$ and $C_{\mathrm{R} \mu}$ fields are

$$
C_{\mathrm{L} \mu} \equiv A_{\mathrm{L} \mu}-\frac{g}{\tilde{g}} \widetilde{W_{\mu}}, \quad C_{\mathrm{R} \mu} \equiv A_{\mathrm{R} \mu}-\frac{g^{\prime}}{\tilde{g}} \widetilde{B_{\mu}}
$$

The $2 \times 2$ matrix $M$ is

$$
M=\frac{1}{\sqrt{2}}\left[v+H+2 i \pi^{a} T^{a}\right], \quad a=1,2,3
$$


where $\pi^{a}$ are the Goldstone bosons produced in the chiral symmetry breaking, $v=\mu / \sqrt{\lambda}$ is the corresponding VEV, $H$ is the composite Higgs, and $T^{a}=\sigma^{a} / 2$, where $\sigma^{a}$ are the Pauli matrices. The covariant derivative is

$$
D_{\mu} M=\partial_{\mu} M-i g \widetilde{W}_{\mu}^{a} T^{a} M+i g^{\prime} M \widetilde{B}_{\mu} T^{3} .
$$

When $M$ acquires its VEV, the Lagrangian of Eq. (B1) contains mixing matrices for the spin one fields. The mass eigenstates are the ordinary SM bosons, and two triplets of heavy mesons, of which the lighter (heavier) ones are denoted by $R_{1}^{ \pm}\left(R_{2}^{ \pm}\right)$and $R_{1}^{0}\left(R_{2}^{0}\right)$. Besides the new heavy leptons, these heavy mesons are the only new particles, at low energy, relative to the SM.

The main input parameters in our implementation of the (N)MWT models are

$$
M_{Z}, G_{F}, e, S, M_{A}, \tilde{g},
$$

where the latter three are parameters of the Technicolor sector per se. The $S$ parameter we fix to be $S=0.1,0.3$ MWT and NMWT models respectively from an estimate based on the underlying dynamics. We then have still the overall mass scale and coupling for the heavy vectors $M_{A}, \tilde{g}$ to scan over. The mass mixing in the neutral sector between the gauge eigenvectors $B, \widetilde{W}, A, V$ is then diagonalized by a $4 \times 4$ orthogonal matrix with entries $N_{i j}\left(\tilde{g}, M_{A}\right)$

$$
\begin{aligned}
B & =N_{11} A+N_{12} Z+N_{13} R_{1}^{0}+N_{14} R_{2}^{0} \\
\widetilde{W}^{3} & =N_{21} A+N_{22} Z+N_{23} R_{1}^{0}+N_{24} R_{2}^{0} \\
A^{3} & =N_{31} A+N_{32} Z+N_{33} R_{1}^{0}+N_{34} R_{2}^{0} \\
V^{3} & =N_{41} A+N_{42} Z+N_{43} R_{1}^{0}+N_{44} R_{2}^{0} .
\end{aligned}
$$

In the limit $\tilde{g} \rightarrow \infty$ we have $N_{33}, N_{44} \rightarrow 1$ while $N_{i j}, i, j=1,2$ take their SM values and all other coefficients go to zero. Upon diagonalization, we organize the mass eigenstates $R_{i}$ such that $M_{R_{1}}<M_{R_{2}}$. The first Weinberg Sum Rule is imposed over the massive vector states. The second Weinberg sum rule is assumed to be modified due to the walking behaviour of the underlying gauge theory. 

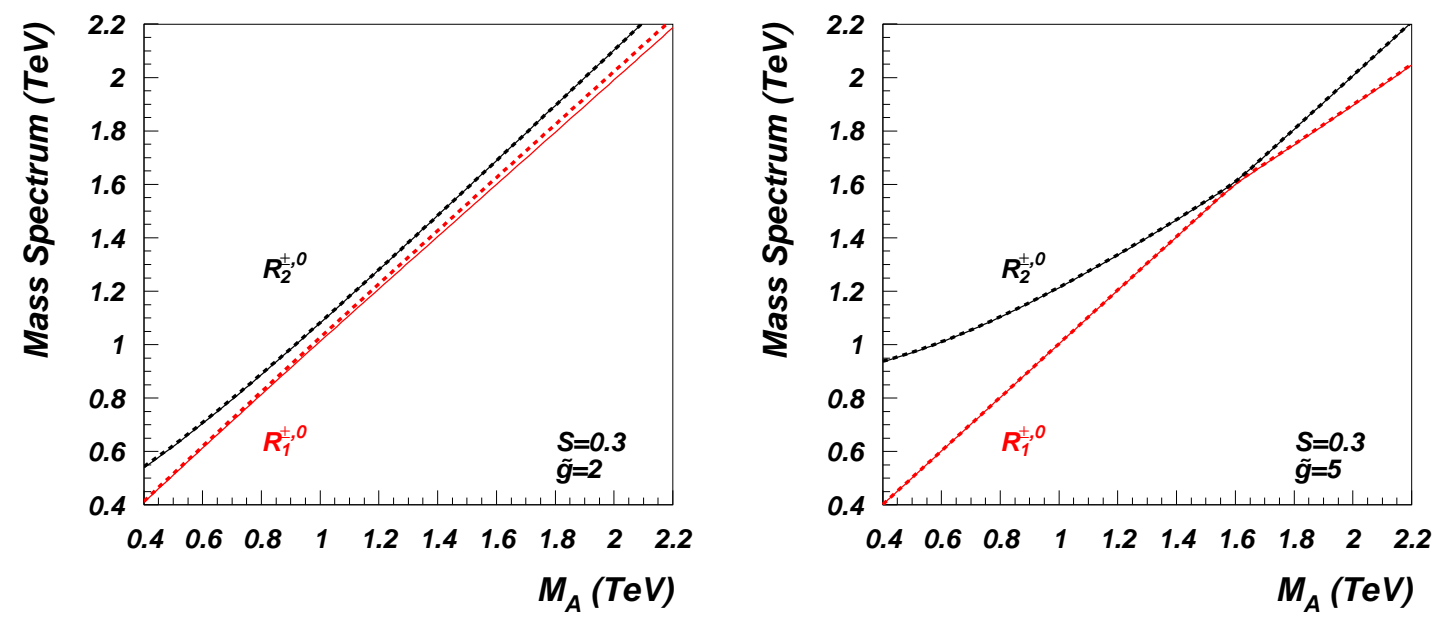

FIG. 16: The mass spectrum of the $M_{R_{1,2}^{ \pm 0}}$ vector mesons versus $M_{A}$ for $\tilde{g}=2$ (left) and $\tilde{g}=5$ (right). The masses of the charged vector mesons are denoted by solid lines, while the masses of the neutral mesons are denoted by dashed lines.

Consequently the mass spectrum and the mixing coefficients are fixed in terms $M_{A}, \tilde{g}$ [5, 37]. We reproduce the plots from the latter reference below: For $\tilde{g}=5$ the inversion point around $1.6 \mathrm{TeV}$ below which $R_{1}$ is (mostly) axial while above it $R_{2}$ is mostly axial (as in QCD) is visible. For $\tilde{g}=2$ where the mixing due to electroweak effects the inversion point is not visible but still occurs in the pure TC sector for $g, g^{\prime}=0$ and still affects processes as detailed in [5, 37]. For $S=0.1$ the inversion point happens at a considerably larger value of $M_{A} \sim 2.5 \mathrm{TeV}$.

In this study we will focus on the parameter values $S=0.1, M_{A}=500$ and we will take two values of the coupling $\tilde{g}=2,5$. The physical parameters with these choices are

$$
\begin{gathered}
M_{R_{1}}=503 \mathrm{GeV}, M_{R_{2}}=996 \mathrm{GeV}, \Gamma\left[R_{1}\right]=0.2 \mathrm{GeV}, \Gamma\left[R_{2}\right]=296 \mathrm{GeV} \\
M_{R_{1}}=518 \mathrm{GeV}, M_{R_{2}}=591 \mathrm{GeV}, \Gamma\left[R_{1}\right]=1 \mathrm{GeV}, \Gamma\left[R_{2}\right]=4 \mathrm{GeV}
\end{gathered}
$$

\section{APPENDIX C: EW PARAMETERS}

The S,T and U parameters [17] in our setup, for $M_{\zeta}, M_{N}>M_{Z}$ for a lepton doublet and additional right-handed singlet as computed in [18]. We keep the hypercharge 
dependence explicit.

$$
\begin{aligned}
S= & \frac{1}{6 \pi}\left\{-Y\left(c_{\theta}^{2} \ln \frac{M_{1}^{2}}{M_{\zeta}^{2}}+s_{\theta}^{2} \ln \frac{M_{2}^{2}}{M_{\zeta}^{2}}\right)+\frac{3}{2}\right. \\
- & \left.s_{\theta}^{2} c_{\theta}^{2}\left(\frac{8}{3}+f_{1}\left(M_{1}, M_{2}\right)-f_{2}\left(M_{1}, M_{2}\right) \ln \frac{M_{1}^{2}}{M_{2}^{2}}\right)\right\} \\
T= & \frac{1}{16 \pi s_{W}^{2} c_{W}^{2} M_{Z}^{2}} \times \\
& \left\{c_{\theta}^{2}\left(M_{1}^{2}+M_{\zeta}^{2}-\frac{2 M_{1}^{2} M_{\zeta}^{2}}{M_{1}^{2}-M_{\zeta}^{2}} \ln \frac{M_{1}^{2}}{M_{\zeta}^{2}}\right)+s_{\theta}^{2}\left(M_{2}^{2}+M_{\zeta}^{2}-\frac{2 M_{2}^{2} M_{\zeta}^{2}}{M_{2}^{2}-M_{\zeta}^{2}} \ln \frac{M_{2}^{2}}{M_{\zeta}^{2}}\right)\right. \\
- & \left.s_{\theta}^{2} c_{\theta}^{2}\left(M_{1}^{2}+M_{2}^{2}-4 M_{1} M_{2}+2 \frac{M_{1}^{3} M_{2}-M_{1}^{2} M_{2}^{2}+M_{1} M_{2}^{3}}{M_{1}^{2}-M_{2}^{2}} \ln \frac{M_{1}^{2}}{M_{2}^{2}}\right)\right\}
\end{aligned}
$$

The functions $f_{1}$ and $f_{2}$ are given by

$$
\begin{aligned}
& f_{1}\left(M_{1}, M_{2}\right)=\frac{3 M_{1} M_{2}^{3}+3 M_{1}^{3} M_{2}-4 M_{1}^{2} M_{2}^{2}}{\left(M_{1}^{2}-M_{2}^{2}\right)^{2}} \\
& f_{2}\left(M_{1}, M_{2}\right)=\frac{M_{1}^{6}-3 M_{1}^{4} M_{2}^{2}+6 M_{1}^{3} M_{2}^{3}-3 M_{1}^{2} M_{2}^{4}+M_{2}^{6}}{\left(M_{1}^{2}-M_{2}^{2}\right)^{3}}
\end{aligned}
$$




\section{APPENDIX D: DECAY WIDTHS OF THE HEAVY LEPTONS}

Decays of the heavy leptons The partial widths of $N_{2}$ before including any mixing with the SM leptons are given by.

$$
\begin{aligned}
\Gamma\left[N_{2} \rightarrow W^{ \pm} \zeta^{\mp}\right] & =\frac{e^{2} s^{2} c^{\prime 2}}{64 \pi s_{w}^{2} M_{2}^{3}} \sqrt{M_{2}^{4}-2 M_{2}^{2} M_{+}^{2}+M_{-}^{4}} \\
& \times\left[\left(M_{2}^{2}+M_{-}^{2}\right)+\frac{\left(M_{2}^{2}-M_{-}^{2}\right)\left(M_{2}^{2}-M_{+}^{2}\right)}{M_{W}^{2}}\right] \\
\Gamma\left[N_{1} \rightarrow W^{ \pm} \zeta^{\mp}\right] & =\frac{e^{2} c^{2} c^{2}}{64 \pi s_{w}^{2} M_{1}^{3}} \sqrt{M_{1}^{4}-2 M_{1}^{2} M_{+}^{2}+M_{-}^{4}} \\
& \times\left[\left(M_{1}^{2}+M_{-}^{2}\right)+\frac{\left(M_{1}^{2}-M_{-}^{2}\right)\left(M_{1}^{2}-M_{+}^{2}\right)}{M_{W}^{2}}\right] \\
\Gamma\left[\zeta^{ \pm} \rightarrow W^{ \pm} N_{1}\right] & =\frac{e^{2} c^{2} c^{\prime 2}}{64 \pi s_{w}^{2} M_{\zeta}^{3}} \sqrt{M_{\zeta}^{4}-2 M_{\zeta}^{2} M_{+}^{\prime 2}+M_{-}^{\prime 4}} \\
& \times\left[\left(M_{\zeta}^{2}+M_{-}^{\prime 2}\right)+\frac{\left(M_{\zeta}^{2}-M_{-}^{\prime 2}\right)\left(M_{\zeta}^{2}-M_{+}^{\prime 2}\right)}{M_{W}^{2}}\right] \\
\Gamma\left[\zeta^{ \pm} \rightarrow W^{ \pm} N_{2}\right] & =\frac{e^{2} s^{2} c^{\prime 2}}{64 \pi s_{w}^{2} M_{\zeta}^{3}} \sqrt{M_{\zeta}^{4}-2 M_{\zeta}^{2} M_{+}^{\prime \prime 2}+M_{-}^{\prime \prime \prime}} \\
& \times\left[\left(M_{\zeta}^{2}+M_{-}^{\prime \prime \prime}\right)+\frac{\left(M_{\zeta}^{2}-M_{-}^{\prime \prime \prime}\right)\left(M_{\zeta}^{2}-M_{+}^{\prime \prime \prime}\right)}{M_{W}^{2}}\right] \\
\Gamma\left[N_{2} \rightarrow Z N_{1}\right] & =\frac{e^{2} c^{2} s^{2}}{64 \pi s_{w}^{2} c_{w}^{2} M_{2}^{3}} \sqrt{M_{2}^{4}-2 M_{2}^{2} M_{+}^{\prime 2}+M_{-}^{\prime 4}} \\
& \times\left[\left(M_{2}^{2}+M_{-}^{\prime 2}\right)+\frac{\left(M_{2}^{2}-M_{-}^{\prime 2}\right)\left(M_{2}^{2}-M_{+}^{\prime 2}\right)}{M_{Z}^{2}}-6 M_{1} M_{2}\right] \\
& \times\left[\left(M_{2}^{2}+M_{-}^{\prime \prime 2}\right)-2 M_{1} M_{2}\right] \\
\Gamma\left[N_{2} \rightarrow H N_{1}\right] & =\frac{e^{2}\left(c^{2}-s^{2}\right)^{2} c^{\prime 2} m_{D}^{2}}{64 \pi s_{w}^{2} M_{W}^{2} M_{2}^{3}} \sqrt{M_{2}^{4}-2 M_{2}^{2} M_{+}^{\prime \prime 2}+M_{-}^{\prime \prime 4}} \\
&
\end{aligned}
$$


where $M_{ \pm}^{2}=M_{\zeta}^{2} \pm M_{W^{\prime}}^{2}, M_{ \pm}^{\prime 2}=M_{1} \pm M_{W^{\prime}}^{2}, M_{ \pm}^{\prime \prime 2}=M_{1} \pm M_{H^{\prime}}^{2}, M_{ \pm}^{\prime \prime \prime 2}=M_{2} \pm M_{W}^{2}$. The additional decays of $N_{1,2}$ induced by the mixing are

$$
\begin{aligned}
\Gamma\left[N_{2} \rightarrow W^{ \pm} \ell^{\mp}\right] & =\frac{e^{2} s^{2} s^{\prime 2} M_{2}^{3}}{64 \pi s_{w}^{2} M_{W}^{2}}\left(1-\frac{M_{W}^{2}}{M_{2}^{2}}\right)\left(1+\frac{M_{W}^{2}}{M_{2}^{2}}-2 \frac{M_{W}^{4}}{M_{2}^{4}}\right) \\
\Gamma\left[N_{1} \rightarrow W^{ \pm} \ell^{\mp}\right] & =\frac{e^{2} c^{2} s^{\prime 2} M_{1}^{3}}{64 \pi s_{w}^{2} M_{W}^{2}}\left(1-\frac{M_{W}^{2}}{M_{1}^{2}}\right)\left(1+\frac{M_{W}^{2}}{M_{1}^{2}}-2 \frac{M_{W}^{4}}{M_{1}^{4}}\right) \\
\Gamma\left[\zeta^{ \pm} \rightarrow W^{ \pm} N_{0}\right] & =\frac{e^{2} s^{\prime 2} M_{\zeta}^{3}}{64 \pi s_{w}^{2} M_{W}^{2}}\left(1-\frac{M_{W}^{2}}{M_{\zeta}^{2}}\right)\left(1+\frac{M_{W}^{2}}{M_{\zeta}^{2}}-2 \frac{M_{W}^{4}}{M_{\zeta}^{4}}\right) \\
\Gamma\left[N_{1} \rightarrow H N_{0}\right] & =\frac{e^{2} s^{2} s^{\prime 2} m_{D}^{2}}{64 \pi s_{w}^{2} M_{W}^{2} M_{1}^{3}}\left(M_{1}^{2}-M_{H}^{2}\right)^{2}
\end{aligned}
$$

The partial widths of the Higgs into the 4 th family are given by

$$
\begin{gathered}
\Gamma\left[H \rightarrow \zeta^{+} \zeta^{-}\right]=\frac{e^{2} m_{\zeta}^{2}}{32 \pi s_{w}^{2} M_{W}^{2}}\left(1-\frac{4 m_{\zeta}^{2}}{M_{H}^{2}}\right) \sqrt{1-\frac{4 m_{\zeta}^{2}}{M_{H}^{2}}} \\
\Gamma\left[H \rightarrow N_{i} N_{i}\right]=\frac{e^{2} c^{2} s^{2} m_{D}^{2}}{16 \pi s_{w}^{2} M_{W}^{2}}\left(1-\frac{4 M_{i}^{2}}{M_{H}^{2}}\right) \sqrt{1-\frac{4 M_{i}^{2}}{M_{H}^{2}}}
\end{gathered}
$$

[1] F. Sannino and K. Tuominen, Phys. Rev. D 71, 051901 (2005) [arXiv:hep-ph/0405209].

[2] C. Amsler et al. [Particle Data Group], "Review of particle physics," Phys. Lett. B 667, 1 (2008).

[3] E. Witten, Phys. Lett. B 117, 324 (1982).

[4] D. D. Dietrich, F. Sannino and K. Tuominen, Phys. Rev. D 72, 055001 (2005) [arXiv:hepph/0505059].

[5] R. Foadi, M. T. Frandsen, T. A. Ryttov and F. Sannino, Phys. Rev. D 76, 055005 (2007) arXiv:0706.1696[hep-ph]].

[6] D. D. Dietrich and M. Jarvinen, Phys. Rev. D 79, 057903 (2009) [arXiv:0901.3528 [hep-ph]].

[7] T. A. Ryttov and F. Sannino, Phys. Rev. D 78, 115010 (2008) [arXiv:0809.0713 [hep-ph]].

[8] T. Appelquist, P. S. Rodrigues da Silva and F. Sannino, "Enhanced global symmetries and the 
chiral phase transition," Phys. Rev. D 60, 116007 (1999) [arXiv:hep-ph/9906555].

[9] P. Achard et al. [L3 Collaboration], Phys. Lett. B 517, 75 (2001) |arXiv:hep-ex/0107015|.

[10] A. Atre, T. Han, S. Pascoli and B. Zhang, arXiv:0901.3589 [hep-ph].

[11] P. Abreu et al. [DELPHI Collaboration], Phys. Lett. B 274, 230 (1992).

[12] T. Aaltonen et al. [CDF Collaboration], arXiv:0902.1266 [hep-ex].

[13] C. Jarlskog, Phys. Lett. B 241 (1990) 579.

[14] E. Nardi, E. Roulet and D. Tommasini, Nucl. Phys. B 386, 239 (1992). E. Nardi, E. Roulet and D. Tommasini, Phys. Lett. B 327, 319 (1994) [arXiv:hep-ph/9402224]. D. Tommasini, G. Barenboim, J. Bernabeu and C. Jarlskog, Nucl. Phys. B 444, 451 (1995) |arXiv:hep-ph/9503228|.

[15] P. Langacker and D. London, Phys. Rev. D 38, 886 (1988).

[16] A. Pich, Nucl. Phys. Proc. Suppl. 181-182, 300 (2008) [arXiv:0806.2793 [hep-ph]].

[17] M. E. Peskin and T. Takeuchi, Phys. Rev. D 46, 381 (1992).

[18] E. Gates and J. Terning, Phys. Rev. Lett. 67, 1840 (1991).

[19] R. Sundrum and S. D. H. Hsu, Nucl. Phys. B 391, 127 (1993) [arXiv:hep-ph/9206225].

[20] T. Appelquist and F. Sannino, Phys. Rev. D 59, 067702 (1999) [arXiv:hep-ph/9806409].

[21] M. Kurachi and R. Shrock, "Behavior of the S parameter in the crossover region between walking and QCD-like regimes of an SU(N) gauge theory," Phys. Rev. D 74, 056003 (2006) |arXiv:hep-ph/0607231|.

[22] [ALEPH Collaboration and DELPHI Collaboration and L3 Collaboration and ], Phys. Rept. 427, 257 (2006) [arXiv:hep-ex/0509008].

[23] D. D. Dietrich, F. Sannino and K. Tuominen, Phys. Rev. D 73, 037701 (2006) [arXiv:hepph/0510217].

[24] B. Holdom, Phys. Rev. D 54, 721 (1996) [arXiv:hep-ph/9602248].

[25] G. Rybka and P. Fisher, arXiv:hep-ex/0507086.

[26] K. Kainulainen, K. Tuominen and J. Virkajarvi, Phys. Rev. D 75, 085003 (2007) [arXiv:hepph/0612247].

[27] C. Kouvaris, Phys. Rev. D 76, 015011 (2007) [arXiv:hep-ph/0703266].

[28] S. B. Gudnason, C. Kouvaris and F. Sannino, Phys. Rev. D 73, 115003 (2006) [arXiv:hepph/0603014].

[29] S. B. Gudnason, C. Kouvaris and F. Sannino, Phys. Rev. D 74, 095008 (2006) arXiv:hepph/0608055]. 
[30] E. Nardi, F. Sannino and A. Strumia, JCAP 0901, 043 (2009) [arXiv:0811.4153 [hep-ph]].

[31] M. Sher and Y. Yuan, Phys. Lett. B 285, 336 (1992).

[32] J. R. Ellis, D. V. Nanopoulos and S. Sarkar, Nucl. Phys. B 259, 175 (1985).

[33] J. R. Ellis, K. Enqvist, D. V. Nanopoulos and S. Sarkar, Phys. Lett. B 167, 457 (1986).

[34] J. R. Ellis, G. B. Gelmini, J. L. Lopez, D. V. Nanopoulos and S. Sarkar, Nucl. Phys. B 373, 399 (1992).

[35] E. Holtmann, M. Kawasaki, K. Kohri and T. Moroi, Phys. Rev. D 60, 023506 (1999) [arXiv:hepph/9805405].

[36] P. H. Frampton, P. Q. Hung and M. Sher, Phys. Rept. 330, 263 (2000) [arXiv:hep-ph/9903387].

[37] A. Belyaev, R. Foadi, M. T. Frandsen, M. Jarvinen, F. Sannino and A. Pukhov, arXiv:0809.0793 [hep-ph].

[38] A. Pukhov, arXiv:hep-ph/0412191.

[39] A. Semenov, arXiv:0805.0555[hep-ph].

[40] T. Cuhadar-Donszelmann, M. K. Unel, V. E. Ozcan, S. Sultansoy and G. Unel, JHEP 0810, 074 (2008) [arXiv:0806.4003 [hep-ph]].

[41] G. D. Kribs, T. Plehn, M. Spannowsky and T. M. P. Tait, Phys. Rev. D 76, 075016 (2007) arXiv:0706.3718 [hep-ph]].

[42] B. Holdom, W. S. Hou, T. Hurth, M. L. Mangano, S. Sultansoy and G. Unel, arXiv:0904.4698 [hep-ph].

[43] A. R. Zerwekh, Eur. Phys. J. C 46, 791 (2006) [arXiv:hep-ph/0512261].

[44] L. Basso, A. Belyaev, S. Moretti and C. H. Shepherd-Themistocleous, arXiv:0812.4313 [hepph].

[45] G. Abbiendi et al. [OPAL Collaboration], Eur. Phys. J. C 49, 457 (2007) [arXiv:hep-ex/0610056].

G. Abbiendi et al. [OPAL Collaboration], arXiv:0707.0373 [hep-ex]. [LEP working group for Higgs boson searches], "Searches for invisible Higgs bosons” arXiv:hep-ex/0107032.

[46] R. Foadi, M. T. Frandsen and F. Sannino, arXiv:0812.3406 [hep-ph].

[47] F. Meisel, M. Duhrssen, M.Heldmann, K. Jacobs, ATL-PHYS-PUB-2006-009,

[48] P. Gagnon, ATL-PHYS-PUB-2005-011.

[49] R. M. Godbole, M. Guchait, K. Mazumdar, S. Moretti and D. P. Roy, Phys. Lett. B 571, 184 (2003) [arXiv:hep-ph/0304137].

[50] H. Davoudiasl, T. Han and H. E. Logan, Phys. Rev. D 71, 115007 (2005) arXiv:hep-ph/0412269]. 
[51] M. Fairbairn, A. C. Kraan, D. A. Milstead, T. Sjostrand, P. Skands and T. Sloan, Phys. Rept. 438, 1 (2007) [arXiv:hep-ph/0611040].

[52] B. C. Allanach, C. M. Harris, M. A. Parker, P. Richardson and B. R. Webber, JHEP 0108, 051 (2001) [arXiv:hep-ph/0108097].

[53] S. Giagu [ATLAS Collaboration and CMS Collaboration], arXiv:0810.1453 [hep-ex]. The CMS Collaboration, CMS PAS EXO-08-003 (2008).

[54] F. del Aguila, J. A. Aguilar-Saavedra and R. Pittau, JHEP 0710, 047 (2007) arXiv:hepph/0703261].

[55] T. Han and B. Zhang, Phys. Rev. Lett. 97, 171804 (2006) |arXiv:hep-ph/0604064].

[56] F. del Aguila and J. A. Aguilar-Saavedra, JHEP 0505, 026 (2005) [arXiv:hep-ph/0503026].

[57] F. del Aguila, J. A. Aguilar-Saavedra and R. Pittau, J. Phys. Conf. Ser. 53, 506 (2006) [arXiv:hepph/0606198].

[58] F. del Aguila and J. A. Aguilar-Saavedra, JHEP 0711, 072 (2007) [arXiv:0705.4117 [hep-ph]].

[59] A. Abulencia et al. [CDF Collaboration], Phys. Rev. Lett. 98, 221803 (2007) [arXiv:hepex/0702051.

[60] T. Aaltonen et al. [CDF Collaboration], Phys. Rev. D 79, 011101 (2009) [arXiv:0809.3781 [hepex]].

[61] R. Foadi, M. Jarvinen and F. Sannino, arXiv:0811.3719 [hep-ph].

[62] R. Foadi and F. Sannino, Phys. Rev. D 78, 037701 (2008) [arXiv:0801.0663 [hep-ph]].

[63] R. Foadi, M. T. Frandsen and F. Sannino, Phys. Rev. D 77, 097702 (2008) [arXiv:0712.1948 [hep-ph]].

[64] S. B. Gudnason, T. A. Ryttov and F. Sannino, Phys. Rev. D 76, 015005 (2007) arXiv:hepph/0612230].

[65] O. Antipin, M. Heikinheimo and K. Tuominen, arXiv:0905.0622 [hep-ph].

[66] The neutral current is not flavor diagonal in models with $\mathrm{TeV}$ scale right handed neutrinos involved in the see-saw mechanism for the light SM neutrino masses, see e.g. [56] 